\title{
Abel's Theorem in the Noncommutative Case
}

\section{FRANK LEITENBERGER}

Fachbereich Mathematik, Universität Rostock, Rostock, D-18051, Germany.

e-mail: frank.leitenberger@mathematik.uni-rostock.de

short title: Abel's Theorem

\begin{abstract}
We define noncommutative binary forms. Using the typical representation of Hermite we prove the fundamental theorem of algebra and we derive a noncommutative Cardano formula for cubic forms. We define quantized elliptic and hyperelliptic differentials of the first kind. Following Abel we prove Abel's Theorem.
\end{abstract}

\section{Introduction}

Finding quantum deformations of the basic objects of algebraic geometry is an open problem. There are different approaches (cf. [11, 14]). Plane curves with genus $g \geq 1$ are related to elliptic and Abelian integrals. Addition theorems of these functions are the content of Abel's theorem. Our approach is based on classical invariant theory and Abel's ideas. We demonstrate that a $h$-deformation has no influence on the validity of Abel's theorem for hyperelliptic differentials of the first kind. For this purpose we develop a theory of a $h$-deformation of the classical invariant theory of binary forms.

Let $C$ be a hyperelliptic curve of genus $g$ with Weierstrass points $a_{1}, \cdots, a_{2 g+2}$, i.e. $C=\left\{(t, u) \in \mathbb{C}^{2} \mid f(t, u)=0\right\} \cup\{\infty\}$, where $f(t, u)=u^{2}-p(t)=u^{2}-\left(t-a_{1}\right) \ldots \ldots\left(t-a_{2 g+2}\right)$. We consider $k$ points $\left(t_{1}, u_{1}\right), \ldots,\left(t_{k}, u_{k}\right)$ on $C$ and form the sum of complex integrals

$$
S=\int_{\infty}^{t_{1}, u_{1}} \frac{v(t) d t}{u}+\int_{\infty}^{t_{2}, u_{2}} \frac{v(t) d t}{u}+\ldots+\int_{\infty}^{t_{k}, u_{k}} \frac{v(t) d t}{u}
$$

where $v(t)$ is a polynomial of degree $\leq g-1$ and a complex path from $\infty$ to $\left(t_{i}, u_{i}\right)$ is chosen in some way. We call $\frac{v(t) d t}{u}$ a hyperelliptic differential of the first kind. We consider on $C$ the meromorphic function

$$
q\left(t, u, c_{1}, \ldots, c_{m_{1}}, d_{1}, \ldots, d_{m_{2}}\right)=a\left(t, c_{1}, \ldots, c_{m_{1}}\right) u-b\left(t, d_{1}, \ldots, d_{m_{2}}\right)
$$

with polynomials $a(t), b(t)$ depending rationally on parameters $c_{i}, d_{i}$. Abel's theorem tells us that $S=0=$ const if the $\left(t_{1}, u_{1}\right), \ldots,\left(t_{k}, u_{k}\right)$ are the points of intersection of $f(t, u)=0$ with the variable algebraic curve $q\left(t, u, c_{i}, d_{i}\right)=0$. We remark that due to $u^{2}-p(t) \equiv 0$ on $C \backslash\{\infty\}$ we can reduce the intersections of any variable algebraic curve $\tilde{q}\left(t, u, \tilde{c_{1}}, \ldots, \tilde{c_{m}}\right)=0$ depending rationally on the $\tilde{c}_{i}$ to the above situation.

Abel's theorem has the differential form

$$
\epsilon_{1} \frac{v\left(t_{1}\right) d t_{1}}{\sqrt{p\left(t_{1}\right)}}+\epsilon_{2} \frac{v\left(t_{2}\right) d t_{2}}{\sqrt{p\left(t_{2}\right)}}+\ldots+\epsilon_{k} \frac{v\left(t_{k}\right) d t_{k}}{\sqrt{p\left(t_{k}\right)}}=0
$$


with $\epsilon_{i}= \pm 1$.

Now we replace the $t_{i}$ in equation (1) by the quotients of homogeneous coordinates $\frac{x_{i}}{y_{i}}$. Furthermore we multiply all components of equation (1) by a certain common factor (cf. Remark 20 below). We can express the hyperelliptic differentials, which are now endowed by this common factor, by bracket symbols

$$
(i j):=\left|\begin{array}{cc}
x_{i} & x_{j} \\
y_{i} & y_{j}
\end{array}\right|, \quad(i, d j):=\left|\begin{array}{cc}
x_{i} & d x_{j} \\
y_{i} & d y_{j}
\end{array}\right|
$$

introduced by Clebsch. Now the components are $S L(2)$-invariant with respect to the natural action on the $x$-y-plane. Therefore we can consider the differential form of Abel's theorem as a proposition about the sum of certain differential invariants. Clebsch introduced the invariant theoretical descriptions of elliptic integrals in [3, p. 228. Further developments are contained in [2].

Most of the modern treatments about Abel's theorem use the Riemanian ideas. For our noncommutative generalization of the differential form (11) we will follow the original proof of Abel (cf. [1]). Abel reduced equation (11) to an algebraic identity, which is a consequence of an expansion into partial fractions (cf. [22, p.28).

We introduce an algebra $H_{I}$ of noncommutative homogeneous coordinates of points of a noncommutative line. The quantum group $U_{h}(s l(2))$ plays the role of the CayleyAronhold differential operators of the classical invariant theory which generate a Lie Algebra $s l(2)$. Because of the Ore property of $H_{I}$ we can extend this algebra to a division algebra.

We define $h$-deformed bracket symbols by $(i j)=x_{i} y_{j}-y_{i} x_{j}-h y_{i} y_{j}$. The classical invariant theory is essentially determined by the algebra of symbols $(i j)$. We make the observation that the algebra of symbols is isomorphic to the classical case. Furthermore the two fundamental theorems of invariant theory are valid.

We define $n$-forms and invariants of $n$-forms. It turns out that the Clebsch Gordan symbolic method works in our situation. By computer calculations we derive the simplest invariants and covariants of quadratic and cubic forms. We use polarization operators in order to replace the independent variables of $n$-forms by the coordinates of arbitrary noncommutative points.

Using the theory of the typical representation of Hermite (cf. [7]) we derive the fundamental theorem of algebra for noncommutative $n$-forms, i.e. we decompose $n$-forms into $n$ commuting linear forms in a certain skew field extension. We describe explizite decompositions for $n=2,3$.

In order to prove Abel's theorem we combine the ideas of Abel and Clebsch. We define elliptic and hyperelliptic differentials as differential invariants in certain differential modules. The addition theorem for elliptic differentials and Abel's theorem for hyperelliptic differentials appear as identities in these differential modules. The classical set of intersection points $\left(t_{i}, u_{i}\right)$ is replaced by a set $\left(X_{i}, Y_{i}\right)$ of homogeneous coordinates of the zeros of a noncommutative $k$-form $r$, which corresponds to an elimination expression $r(t)$ of $f(t, u)$ and $q\left(t, u, c_{i}, d_{i}\right)$ in the classical case. The analogy of the proof to the classical case is based on the fact that the elements $(i j), y_{k}, d z_{l}$ commute. The $h$-deformation has no influence over any step of the classical invariant theoretical proof.

We used the computer algebra program Mathematica 3.0 to form the computations.

\section{Preliminaries}


In this section we introduce some basic concepts about the quantum group $U_{h}(\operatorname{sl}(2))$ and the braided module algebras $H_{I}$. We follow the similar considerations in [12] for the quantum group $U_{q}(g l(2))$.

Let $U_{h}(s l(2))$ with $h \in \mathbb{C}, h \neq 0$ be the complex unital Hopf algebra, determined by generators $E, F, H$ and relations

$$
\begin{aligned}
& {[H, E]=E(\cosh (h F))+(\cosh (h F)) E} \\
& {[H, F]=-\frac{2}{h} \sinh (h F)} \\
& {[E, F]=H .}
\end{aligned}
$$

The Hopf multiplication is given by

$$
\begin{aligned}
& \Delta(E)=E \otimes e^{-h F}+e^{h F} \otimes E, \\
& \Delta(F)=F \otimes 1+1 \otimes F, \\
& \Delta(H)=H \otimes e^{-h F}+e^{h F} \otimes H .
\end{aligned}
$$

$U_{h}(\operatorname{sl}(2))$ has the counit

$$
\epsilon(E)=\epsilon(F)=\epsilon(H)=0, \quad \epsilon(1)=1 \text {. }
$$

and the coinverse

$$
S(E)=-e^{h F} E e^{-h F}, \quad S(F)=-F, \quad S(H)=-e^{h F} H e^{-h F} .
$$

For an arbitrary ordered index set $I$ we consider the complex unital $U_{h}(\operatorname{sl}(2))$-module algebra $A_{I}$, which is freely generated by the variables $x_{i}, y_{i}, i \in I$. To determine the action of $U_{h}(s l(2))$ on $A_{I}$, we set

$$
\begin{aligned}
& H 1=0, \quad H x_{i}=x_{i}, \quad H y_{i}=-y_{i}, \\
& E 1=0, \quad E x_{i}=0, \quad E y_{i}=x_{i} \\
& F 1=0, \quad F x_{i}=y_{i}, \quad F y_{i}=0,
\end{aligned}
$$

and require

$$
\begin{array}{lll}
E(a b)=E(a) e^{-h F}(b) & +e^{h F}(a) E(b) \\
F(a b)=F(a) b & +a F(b) \\
H(a b)=H(a) e^{-h F}(b) & +e^{h F}(a) H(b)
\end{array}
$$

for $a, b \in H_{I}$. A proof can be given along the lines of [9], p.19 where a similar module occurs.

Furthermore we consider the ideal $J$ of $A_{I}$, which is generated by the elements

$$
\begin{aligned}
& x_{i} y_{i}-y_{i} x_{i}-h y_{i}^{2}, \\
& x_{j} y_{i}-y_{i} x_{j}-h y_{i} y_{j}, \\
& y_{j} x_{i}-x_{i} y_{j}+h y_{i} y_{j}, \\
& y_{j} y_{i}-y_{i} y_{j}, \\
& x_{j} x_{i}-x_{i} x_{j}+h x_{i} y_{j}-h y_{i} x_{j}-h^{2} y_{i} y_{j}
\end{aligned}
$$

for $i<j$. One can show that the ideal $J$ is $U_{h}(s l(2))$-invariant (i.e. $E(J), F(J), H(J) \subseteq J$ (cf. [13]). Therefore the action of $U_{h}(s l(2))$ on $A_{I}$ induces an action on $H_{I}:=A_{I} / J$. In 
the following we identify $x_{i}$ with its image under the quotient map $A_{I} \rightarrow H_{I}$. I.e. $H_{I}$ is the unital $U_{h}(s l(2))$-module algebra with generators $x_{i}, y_{i} i \in I$ and relations

$$
\begin{array}{ll}
x_{i} y_{i}=y_{i} x_{i}+h y_{i}^{2}, & \\
x_{j} y_{i}=y_{i} x_{j}+h y_{i} y_{j}, & y_{j} x_{i}=x_{i} y_{j}-h y_{i} y_{j}, \\
y_{j} y_{i}=y_{i} y_{j}, & x_{j} x_{i}=x_{i} x_{j}-h x_{i} y_{j}+h y_{i} x_{j}+h^{2} y_{i} y_{j}
\end{array}
$$

for $i<j$.

Remark 1. $H_{I}$ is a braided module algebra (cf. [13]). We have $\left(x_{i}+x_{j}\right)\left(y_{i}+y_{j}\right)=$ $\left(y_{i}+y_{j}\right)\left(x_{i}+x_{j}\right)+h\left(y_{i}+y_{j}\right)\left(y_{i}+y_{j}\right)$. The algebra $H_{I}$ has the PBW property for an arbitrary order of the variables $x_{i}, y_{i}, i \in I$. We can consider the equations (5) as rules in order to express an element $a \in H_{I}$ by the PBW basis $x_{i_{1}}^{n_{1}} y_{i_{1}}^{m_{1}} x_{i_{2}}^{n_{2}} y_{i_{2}}^{m_{2}} \ldots x_{i_{k}}^{n_{k}} y_{i_{k}}^{m_{k}}$ with $i_{1}<i_{2}<\ldots<i_{k}$ and $k \in \mathbb{N}$. The rules (5) do not change the type of homogeneity with respect to the indices $i \in I$.

We note that $H_{I}$ is the only module algebra with quadratic relations which is braided and has the PBW property.

We will extend $H_{I}$ to a division algebra $Q_{I}$. Let $R$ be an algebra, $\sigma$ an endomorphism of $R$ and $\delta$ a $\sigma$-derivation with

$$
\delta(a b)=\delta(a) b+\sigma(a) \delta(b)
$$

We say that $S=R[x ; \sigma, \delta]$ is an Ore extension of $R$ if $S$ is freely generated over $R$ by an element $x$ subject only to the relation

$$
x a=\sigma(a) x+\delta(a)
$$

for $a \in R$ (cf. [15]).

Lemma 1. The algebra $H_{I}$ is an iterated Ore extension.

Proof. We restrict the consideration to the finite generated algebras $H_{I}$ with $I=$ $\{0,1, \ldots, n\}$. In the case of an infinite index set $I$ one can proceed by transfinite induction. We consider the tower of subalgebras

$$
\mathbb{C} \subset H_{0}^{\prime} \subset H_{0} \subset H_{1}^{\prime} \subset \cdots \subset H_{n}^{\prime} \subset H_{n}
$$

where $H_{j}^{\prime} \cong H_{j-1}\left[y_{j}, \sigma, \delta\right]$ is the subalgebra generated by $H_{j-1}$ and $y_{j}$, where $\delta=0$ and $\sigma$ is determined by

$$
\sigma\left(x_{i}\right)=x_{i}-h y_{i}, \quad \sigma\left(y_{i}\right)=y_{i}
$$

for $i<j$. Furthermore we have $H_{j} \cong H_{j}^{\prime}\left[x_{j}, \sigma, \delta\right]$ where $\sigma$ and $\delta$ are determined by

$$
\begin{gathered}
\sigma\left(x_{i}\right)=x_{i}+h y_{i}, \quad \sigma\left(y_{i}\right)=y_{i}, \quad \sigma\left(y_{j}\right)=y_{j}, \\
\delta\left(x_{i}\right)=-h\left(x_{i}-h y_{i}\right) y_{j}, \quad \delta\left(y_{i}\right)=h y_{i} y_{j}, \quad \delta\left(y_{j}\right)=h y_{j}^{2}
\end{gathered}
$$

for $i<j$. The verification of the isomorphies is similar to [8], p. 81. The assertion follows. 
Proposition 2. The algebra $H_{I}$ has no zero divisors and $H_{I}$ has an uniquely determined right quotient division ring $Q_{I}$, whose elements are the right quotients $a b^{-1}$ for $a, b \in R$, $b \neq 0$.

Proof. Ore proved that a noncommutative ring without zero divisors has an uniquely determined quotient division ring if

$$
a R \cap b R \neq\{0\} \quad \forall a, b \in R, \quad a, b \neq 0,
$$

i.e., two nonvanishing elements $a, b$ have a common right multiple (cf. [18]). Curtis proved that an iterated Ore extension of a ring without zero divisors with the property (6) is again a ring without zero divisors with the property (6]) (cf. [5]). The assertion follows from both results together with Lemma 1

Example 1. For $x_{1}$ and $x_{2}$ we find the common right multiple

$$
x_{2}\left(x_{1}-h y_{1}\right)=x_{1}\left(x_{2}-h y_{2}\right) .
$$

Therefore we can represent the left quotient $x_{1}^{-1} x_{2}$ by the right quotient $\left(x_{2}-h y_{2}\right)\left(x_{1}-h y_{1}\right)^{-1}$.

Remark 2. We can consider the variables $x_{i}, y_{i}$ as noncommutative homogeneous coordinates of different points of the complex projective line. The noncommutative coordinate is given by

$$
z_{i}:=x_{i} y_{i}^{-1}+\frac{h}{2} \in Q_{I} .
$$

For an involution $x_{i}^{*}:=x_{i}, y_{i}^{*}:=y_{i}$ and $\operatorname{Re} h=0$ we have $z_{i}^{*}=z_{i}$. This motivates the $\frac{h}{2}$ -term. For two points we have the commutation relation

$$
z_{j} z_{i}=z_{i} z_{j}+2 h\left(z_{j}-z_{i}\right), \quad \forall i, j
$$

or $\left(z_{j}+h\right)\left(z_{i}-h\right)=\left(z_{i}+h\right)\left(z_{j}-h\right)$.

\section{The Invariants of the algebra $H_{I}$.}

Definition 1. We say that the element $v \in H_{I}$ is an invariant element, if

$$
E(v)=F(v)=H(v)=0 .
$$

Remark 3. Alternatively, one can define invariant elements by the Quantum group $S L_{h}(2)$. The algebra structure is given by the generators $a, b, c, d$ and the relations $a b-b a=h\left(1-a^{2}\right), \quad a c-c a=h c^{2}, \quad b d-d b=h\left(d^{2}-1\right)$, $c d-d c=-h c^{2}, \quad a d-d a=h c(d-a), \quad b c-c b=h(d c+c a)$ and $a d-b c+h a c=1$. A coaction $\omega$ of $S L_{h}(2)$ on $H_{I}$ is given by

$$
\left(\begin{array}{l}
\omega(x) \\
\omega(y)
\end{array}\right):=\left(\begin{array}{c}
a \otimes x+b \otimes y \\
c \otimes x+d \otimes y
\end{array}\right) .
$$

Now we can define invariant elements by the equation

$$
\omega(v)=1 \otimes v .
$$


By the rules (2) sums and products of invariant elements are again invariant elements. Therefore they form a subalgebra $H_{I}^{I n v} \subset H_{I}$.

Example 2. The simplest invariant element of $H_{I}$ is given by

$$
(i j):=x_{i} y_{j}-y_{i} x_{j}-h y_{i} y_{j}
$$

We have also $(i j)=x_{i} y_{j}-x_{j} y_{i}=y_{j} x_{i}-y_{i} x_{j}$.

In the following we will see, that the subalgebra $H_{I}^{I n v}$ has many classical properties.

Proposition 3 . The bracket symbols $(i j), i, j \in I$ form a central, commutative subalgebra $S_{I} \subseteq H_{I}^{I n v}$.

Proof: The identities $x_{k}(i j)=(i j) x_{k}, y_{k}(i j)=(i j) y_{k}, i, j, k, \in I$ can be checked by an explizite calculation. Therefore the bracket $(i j)$ is central. In particular we have $(k l)(i j)=(i j)(k l), \forall i, j, k, l \in I$. Therefore $S_{I}$ is commutative.

The bracket symbols $(i j)$ have the following simple properties $(i i)=0$ and

$$
(j i)+(i j)=0, \quad(i j)(k l)+(i k)(l j)+(i l)(j k)=0 \quad \forall i, j, k, l .
$$

The second identity is the Grassmann Plücker relation.

Now we consider the two Fundamental Theorems of invariant theory. We have the First Fundamental Theorem:

Theorem 4. We have $H_{I}^{I n v}=S_{I}$, i.e. the algebra $H_{I}^{I n v}$ is generated by the bracket symbols $(i j)$.

A proof can be given similar to the case of the $U_{q}\left(g l_{2}\right)$-symmetry (cf. [12]).

Because $H_{I}^{I n v}$ is generated by bracket symbols, we can consider $H_{I}^{I n v}$ as a quotient of an algebra $B_{I}$, freely generated by commuting symbols $[i j]$ and an ideal $S$ of syzygies (i.e. $(i j) \cong[i j]+S)$. The Second Fundamental Theorem of classical invariant theory tells us that $S$ is generated by the elements $[i j]+[j i]$ and $[i j][k l]+[i k][l j]+[i l][j k]$ (cf. [19]).

The Second Fundamental Theorem is also valid for $h \neq 0$ :

Theorem 5. Using only arithmetical operations of bracket symbols, every vanishing expression of bracket symbols can be transformed into

$$
\sum_{i j}((i j)+(j i)) I_{i j}+\sum_{i j k l}((i j)(k l)+(i k)(l j)+(i l)(j k)) I_{i j k l}
$$

with certain expressions $I_{i j}, I_{i j k l}$ of bracket symbols.

Proof: For every $h \in \mathbb{C}$ we consider the homomorphism $g_{h}: B_{I} \rightarrow H_{I}^{I n v}=H_{I}^{I n v}(h)$ with $g_{h}([i j])=(i j)$. Let $S_{h}:=\operatorname{Ker} g_{h}$. By the classical Second Fundamental Theorem and (7) we have $S_{0} \subseteq S_{h}$. Conversely, let $e \in B_{I}$. We consider the PBW expansion of $g_{h}(e)$. If we apply the rules (5-5) to a monomial of the $x_{i}, y_{i}$ then the $x_{i}, y_{i}$ permute without changing the coefficient of the monomial and we obtain additional terms with a higher degree in the $y_{i}$. Therefore we have the decomposition

$$
g_{h}(e)=e_{0}^{(h)}+e_{1}^{(h)}
$$

where $e_{0}^{(h)}$ contains all monomials $x_{1}^{n-i_{1}} y_{1}^{i_{1}} x_{2}^{n-i_{2}} y_{2}^{i_{2}} \ldots x_{n}^{n-i_{n}} y_{n}^{i_{k}}$ with $\sum_{j} i_{j}=\frac{1}{2} \sum_{j} n_{j}$ and $e_{1}^{(h)}$ contains all monomials with $\sum_{j} i_{j}>\frac{1}{2} \sum_{j} n_{j}$. The coefficients of $e_{0}^{(h)}$ are independent of $h$ and $e_{1}^{(h)}$ is 0 for $h=0$. Therefore $g_{h}(e)=0$ yields $g_{0}(e)=0$, i.e. $S_{h} \subseteq S_{0}$. 
Corollary 6. An identity between bracket symbols is valid if and only if the identity is valid for $h=0$.

\section{Binary forms}

We use the algebra $H_{I}$ in order to introduce noncommutative binary homogeneous $n$-forms.

For the following we suppose for the algebra $H_{I}$ that $0 \in I$. We will use the short notations

$$
x:=x_{0}, \quad y:=y_{0} .
$$

Furthermore let $H_{I \backslash\{0\}}$ be the subalgebra of $H_{I}$ which is generated by the elements $x_{i}, y_{i}$, $i \in I \backslash\{0\}$.

Definition 2. We say that the nonvanishing element

$$
f:=x^{n} A_{0}+\left(\begin{array}{c}
n \\
1
\end{array}\right) x^{n-1} y A_{1}+\left(\begin{array}{c}
n \\
2
\end{array}\right) x^{n-2} y^{2} A_{2}+\ldots .+y^{n} A_{n}
$$

of $H_{I}$ with $A_{i} \in H_{I \backslash\{0\}}$ is a $n$-form, if $f \in H_{I}^{I n v}$.

Remark 4. The representation of $f$ with coefficients $A_{i} \in H_{I \backslash\{0\}}$ is unique. This is a consequence of the PBW theorem.

Remark 5. Alternatively one can consider left coefficients. For example, for $f=(01)^{2}=$ $x^{2} A+2 x y B+y^{2} C=A_{L} x^{2}+2 B_{L} x y+C_{L} y^{2}$ we have

$$
\begin{aligned}
& A=y_{1}^{2}, \quad B=-x_{1} y_{1}-\frac{h}{2} y_{1}^{2}, \quad C=x_{1}^{2}+3 h x_{1} y_{1}, \\
& A_{L}=y_{1}^{2}, \quad B_{L}=-x_{1} y_{1}+\frac{3 h}{2} y_{1}^{2}, \quad C_{L}=x_{1}^{2}-h x_{1} y_{1} .
\end{aligned}
$$

Proposition 7. Let $f$ be an arbitrary $n$-form. Then the coefficients of $f$ obey the commutation relations of the coefficients of the special $n$-form $(01)(02) \ldots(0 n)$.

Proof: Let $f_{1}=\left(0 i_{1}\right) \ldots\left(0 i_{n}\right)=\sum\left(\begin{array}{c}n \\ i\end{array}\right) x^{i} y^{n-i} A_{i}$ and $f_{2}=\left(0 j_{1}\right) \ldots\left(0 j_{n}\right)=\sum\left(\begin{array}{c}n \\ i\end{array}\right) x^{i} y^{n-i} A^{\prime}{ }_{i}$ be two $n$-forms with $2 n$ different indices $i_{k}, j_{k}$. By (5) we have commutation relations of the form

$$
A^{\prime}{ }_{l} A_{k}-A_{k} A^{\prime}{ }_{l}=\sum_{i \leq k, j \leq l, i+j<k+l} \alpha_{i j} A_{i} A^{\prime}{ }_{j}
$$

Because of the PBW property the $\alpha_{i j}$ are uniquely determined. These relations are also valid if not all $i_{k}, j_{k}$ are different. In the case $f_{1}=f_{2}$ we can simplify the relations to

$$
A_{l} A_{k}-A_{k} A_{l}=\sum_{i \leq j, i \leq k, j \leq l, i+j<k+l} \beta_{i j} A_{i} A_{j},
$$

i.e. $\beta_{i j}=0$ for $i>j$. According to Theorem 4 two arbitrary $n$-forms $f$ and $f^{\prime}$ have the representations

$$
\sum_{i_{1}, \ldots, i_{n} \in I \backslash\{0\}}\left(0 i_{1}\right)\left(0 i_{2}\right) \ldots\left(0 i_{n}\right) P_{i_{1}, \ldots, i_{n}}, \quad \sum_{i_{1}, \ldots, i_{n} \in I \backslash\{0\}}\left(0 i_{1}\right)\left(0 i_{2}\right) \ldots\left(0 i_{n}\right) P_{i_{1}, \ldots, i_{n}}^{\prime}
$$


with central elements $P_{i_{1}, \ldots, i_{n}}, P_{i_{1}, \ldots, i_{n}}^{\prime} \in H_{I \backslash\{0\}}^{I n v}$. Therefore $f$ and $f^{\prime}$ have the coefficients

$$
A_{i}=\sum A_{i}^{i_{1}, \ldots, i_{n}} P^{\prime}{ }_{i_{1}, \ldots, i_{n}}, \quad A^{\prime}{ }_{i}=\sum A_{i}^{i_{1}, \ldots, i_{n}} P^{\prime \prime}{ }_{i_{1}, \ldots, i_{n}}
$$

where $A_{i}^{i_{1}, \ldots, i_{n}}$ are the coefficients of the $n$-form $\left(0 i_{1}\right)\left(0 i_{2}\right) \ldots\left(0 i_{n}\right)$. Therefore the equation (9) is also valid for $f$ and $f^{\prime}$. It follows (10) for $f=f^{\prime}$.

Remark 6. Proposition $\square$ is not true for the quantum group $U_{q}(s l(2))$ (cf. [12]), i.e. the commutation relations depend on the concrete type of form.

Example 3. Consider the quadratic form

$$
f:=(01)(02)=x^{2} A+x y B+y^{2} C
$$

with

$$
A=y_{1} y_{2}, B=-\frac{h}{2}\left(x_{1} y_{2}+y_{1} x_{2}\right)-h y_{1} y_{2}, C=x_{1} x_{2}+h x_{1} y_{2}+2 h y_{1} x_{2}+2 h^{2} y_{1} y_{2} .
$$

For $f$ we have the following commutation relations

$$
\begin{gathered}
C A=A C-4 h A B+6 h^{2} A^{2}, \\
C B=B C-2 h A C+2 h^{2} A B-3 h^{3} A^{2}, \\
B A=A B-2 h A^{2},
\end{gathered}
$$

Example 4. For the cubic form

$$
f=(01)(02)(03)
$$

we have

$$
\begin{aligned}
& A=y_{1} y_{2} y_{3}, \\
& B=-\frac{h}{3}\left(x_{1} y_{2} y_{3}+y_{1} x_{2} y_{3}+y_{1} y_{2} x_{3}\right)-h y_{1} y_{2} y_{3}, \\
& C=\frac{h}{3}\left(x_{1} x_{2} y_{3}+x_{1} y_{2} x_{3}+y_{1} x_{2} x_{3}\right)+\frac{2 h}{3} x_{1} y_{2} y_{3}+h y_{1} x_{2} y_{3}+\frac{4 h}{3} y_{1} y_{2} x_{3}+2 h^{2} y_{1} y_{2} y_{3}, \\
& D=-x_{1} x_{2} x_{3}-h x_{1} x_{2} y_{3}-2 h x_{1} y_{2} x_{3}-3 h y_{1} x_{2} x_{3}-2 h^{2} x_{1} y_{2} y_{3}-3 h^{2} y_{1} x_{2} y_{3}-6 h^{2} y_{1} y_{2} x_{3}-6 h_{3} y_{1} y_{2} y_{3}
\end{aligned}
$$

and the commutation relations

$$
\begin{aligned}
& D A=A D-9 h A C+36 h^{2} A B-60 h^{3} A^{2}, \\
& D B=B D-3 h A D-3 h B C+9 h^{2} A C+6 h^{2} B^{2}-24 h^{3} A B+36 h^{4} A^{2}, \\
& D C=C D-6 h B D+3 h C^{2}+12 h^{2} A D-6 h^{3} A C-18 h^{3} B^{2}+36 h^{4} A B-48 h^{5} A^{2}, \\
& C A=A C-6 h A B+12 h^{2} A^{2}, \\
& C B=B C-h A C-2 h B^{2}+2 h^{2} A B-4 h^{3} A^{2}, \\
& B A=A B-3 h A^{2} .
\end{aligned}
$$

\section{Linear forms and points}


The simplest example of a linear form is

$$
x A+y B:=(01)=x y_{1}-y\left(x_{1}+h y_{1}\right),
$$

i.e. $A=y_{1}, B=-x_{1}-h y_{1}$.

Another example is the form

$$
x A+y B:=(02)(13)+(03)(12)
$$

corresponding to the fourth harmonic point with

$$
\begin{aligned}
& A=2 x_{1} y_{2} y_{3}-y_{1} x_{2} y_{3}-y_{1} y_{2} x_{3}-3 h y_{1} y_{2} y_{3} \\
& B=-x_{1} x_{2} y_{3}-x_{1} y_{2} x_{3}+2 y_{1} x_{2} x_{3}-h x_{1} y_{2} y_{3}+h y_{1} x_{2} y_{3}+3 h y_{1} y_{2} x_{3}+3 h^{2} y_{1} y_{2} y_{3} .
\end{aligned}
$$

Let $f=x A+y B$ be a linear form and let

$$
Y=A, \quad X=-B-h A .
$$

Then $f$ has the representation

$$
f=x Y-y X-h y Y=-(X y-Y x-h Y y) .
$$

Proposition 8. Let $f=x Y-y X-h y Y$ and $\bar{f}=x Y^{\prime}-y X^{\prime}-h y Y^{\prime}$ be two linear forms. The elements $X, Y, X^{\prime}, Y^{\prime}$ have commutation relations similar to (5), i.e. we have

$$
\begin{array}{ll}
X Y=Y X+h Y^{2}, & X^{\prime} Y^{\prime}=Y^{\prime} X^{\prime}+h Y^{\prime 2}, \\
X X^{\prime}=X^{\prime} X-h X^{\prime} Y+h Y^{\prime} X+h^{2} Y^{\prime} Y, & Y Y^{\prime}=Y^{\prime} Y, \\
X Y^{\prime}=Y^{\prime} X+h Y^{\prime} Y, & Y X^{\prime}=X^{\prime} Y-h Y^{\prime} Y .
\end{array}
$$

The proof is analogous to Proposition 7.

Remark 7. We consider $X, Y$ as homogeneous coordinates of a noncommutative point with the projective coordinate $Z:=X Y^{-1}+\frac{h}{2} \in Q_{I}$.

\section{Invariants of Forms}

We consider a $n$-form $f=\sum_{i=0}^{n}\left(\begin{array}{c}n \\ i\end{array}\right) x^{n-i} y^{i} A_{i}$.

Definition 3. We say that the polynomial expression of the coefficients of $f$

$$
I_{f}=I_{f}\left(A_{0}, \ldots, A_{n}\right)
$$

is an invariant, if $I_{f} \in H_{I \backslash\{0\}}^{I n v}$. We say that the polynomial expression

$$
C_{f}=C_{f}\left(A_{0}, \ldots, A_{n}, x_{0}, y_{0}\right)
$$

is a covariant, if $C_{f}$ is a $m$-form.

Example 5. The simplest invariant of a quadratic form is the discriminant $A C-B^{2}-h A B+\frac{3 h^{2}}{4} A^{2}$ (cf. below).

\section{The Symbolic Method}


We consider the $n$-form

$$
f=x^{n} A_{0}+\left(\begin{array}{c}
n \\
1
\end{array}\right) x^{n-1} y A_{1}+\left(\begin{array}{c}
n \\
2
\end{array}\right) x^{n-2} y^{2} A_{2}+\ldots .+y^{n} A_{n} .
$$

Our aim is to construct invariants of degree $k$.

We consider the collection of $k$ very simple $n$-forms

$$
f^{\prime}:=(01)^{n}, \quad f^{\prime \prime}:=(02)^{n}, \quad \ldots, \quad f^{(k)}:=(0 k)^{n} .
$$

Because of (5) the form $f^{(j)}$ has the coefficients $A_{i}^{(j)}$ with

$$
A_{i}^{(j)}=(-1)^{i}\left(\begin{array}{c}
n \\
i
\end{array}\right) x_{j}^{i} y_{j}^{n-i}+\sum_{k=1}^{i} c_{i k} h^{k} x_{j}^{i-k} y_{j}^{n-i+k}
$$

and certain numbers $c_{i k} \in \mathbb{Z}$.

Consider an element $d$ of $H_{I}^{I n v}$ which is homogeneous of degree $n$ with respect to the indices $1,2, \ldots, k$. (For example $(12)(13)(23)$ is homogeneous of degree 2 with respect to 1, 2,3.)

Because of the PBW theorem for $H_{I}$ and because the application of the reduction rules (15) does not change the degree of homogeneity with respect to $1,2, \ldots, k$ we can express $d$ as a linear combination of monomials

$$
x_{1}^{n-i_{1}} y_{1}^{i_{1}} x_{2}^{n-i_{2}} y_{2}^{i_{2}} \ldots x_{k}^{n-i_{k}} y_{k}^{i_{k}}
$$

In these products we replace expressions $x_{j}^{n}$ by

$$
=(-1)^{n}\left(A_{n}^{(j)}-\sum_{k=1}^{n} c_{n k} h^{k} x_{j}^{n-k} y_{j}^{k}\right) .
$$

We obtain monomials of products of terms $x_{j}^{n-i} y_{j}^{i}$ with $1 \leq i \leq n$. Then we replace the products $x_{j}^{n-1} y_{j}^{1}$ by

$$
=(-1)^{n-1}\left(\begin{array}{l}
n \\
1
\end{array}\right)^{-1}\left(A_{n-1}^{(j)}-\sum_{k=1}^{n-1} c_{n-1, k} h^{k} x_{j}^{n-1-k} y_{j}^{1+k}\right),
$$

etc.. In the $n+1$-th step we replace the terms $y_{j}^{n}$ by $A_{0}^{(j)}$.

In this way we obtain for the symbol $d$ the representation

$$
d=\sum c_{i_{1}, i_{2}, \ldots, i_{k}} A_{i_{1}}^{(1)} A_{i_{2}}^{(2)} \ldots A_{i_{k}}^{(k)} .
$$

Furthermore we consider the elements

$$
\mathcal{I}^{\prime}=\sum c_{i_{1}, i_{2}, \ldots, i_{k}} \mathcal{A}_{i_{1}}^{(1)} \mathcal{A}_{i_{2}}^{(2)} \ldots \mathcal{A}_{i_{k}}^{(k)}
$$

for $k$ arbitrary $n$-forms $F^{j}$ with coefficients $\mathcal{A}_{i}^{(j)}$.

We form

$$
E\left(\mathcal{I}^{\prime}\right)=\sum d_{i_{1}, i_{2}, \ldots, i_{k}} \mathcal{A}_{i_{1}}^{(1)} \mathcal{A}_{i_{2}}^{(2)} \ldots \mathcal{A}_{i_{k}}^{(k)}
$$


and consider the homomorphism $h_{f^{\prime}, \ldots, f^{(k)}}$ determined by

$$
h_{f^{\prime}, \ldots, f^{(k)}}\left(\mathcal{A}_{i}^{j}\right):=A_{i}^{j} .
$$

It follows

$$
\begin{gathered}
0=E(d)=E\left(h_{f^{\prime}, \ldots, f^{(k)}}\left(\mathcal{I}^{\prime}\right)\right)=h_{f^{\prime}, \ldots, f^{(k)}}\left(E\left(\mathcal{I}^{\prime}\right)\right)=\sum d_{i_{1}, i_{2}, \ldots, i_{k}} A_{i_{1}}^{(1)} A_{i_{2}}^{(2)} \ldots A_{i_{k}}^{(k)} \\
=\sum_{i_{1}, \ldots, i_{k}} d_{i_{1}, i_{2}, \ldots, i_{k}}(-1)^{i_{1}+\ldots+i_{k}}\left(\left(\begin{array}{c}
n \\
i_{1}
\end{array}\right) \ldots\left(\begin{array}{c}
n \\
i_{k}
\end{array}\right)\right)^{-1} x_{1}^{i_{1}} y_{1}^{n-i_{1}} x_{2}^{i_{2}} y_{2}^{n-i_{2}} \ldots x_{k}^{i_{k}} y_{k}^{n-i_{k}} \\
+\sum_{i_{1}, \ldots, i_{k}} d_{i_{1}, i_{2}, \ldots, i_{k}} \sum_{1 \leq l_{1} \leq i_{1}, \ldots, 1 \leq l_{k} \leq i_{k}} c_{i_{1} l_{1} \ldots} c_{i_{k} l_{k}} h^{l_{1}+\ldots+l_{k}} x_{1}^{i_{1}-l_{1}} y_{1}^{n-i_{1}+l_{1}} x_{2}^{i_{2}-l_{2}} y_{2}^{n-i_{2}+l_{2}} \ldots x_{k}^{i_{k}-l_{k}} y_{k}^{n-i_{k}+l_{k}}
\end{gathered}
$$

The PBW theorem allows a comparison of coefficients. For the coefficient at $x_{1}^{i_{1}} y_{1}^{n-i_{1}} x_{2}^{i_{2}} y_{2}^{n-i_{2}} \ldots x_{k}^{i_{k}} y_{k}^{n-i_{k}}$ we have

$$
\begin{array}{r}
0=d_{i_{1}, i_{2}, \ldots, i_{k}}(-1)^{i_{1}+\ldots+i_{k}}\left(\left(\begin{array}{l}
n \\
i_{1}
\end{array}\right) \ldots\left(\begin{array}{l}
n \\
i_{k}
\end{array}\right)\right)^{-1}+ \\
+\sum_{1 \leq l_{1} \leq n-i_{1}, \ldots, 1 \leq l_{k} \leq n-i_{k}} d_{i_{1}+l_{1}, \ldots, i_{k}+l_{k}} c_{i_{1}+l_{1}, l_{1} \ldots c_{i_{k}+l_{k} l_{k}} h^{l_{1}+\ldots+l_{k}}}
\end{array}
$$

These equations form a triangular equation system for the elements $d_{i_{1}, \ldots, i_{k}}$ with the unique solution $d_{i_{1}, \ldots, i_{k}}=0$, i.e. $E\left(\mathcal{I}^{\prime}\right)=0$. Similarly we obtain $F\left(\mathcal{I}^{\prime}\right)=0$ and $H\left(\mathcal{I}^{\prime}\right)=0$. Therefore $\mathcal{I}^{\prime}$ is a common invariant of $k$ arbitrary $n$-forms $F^{\prime}, F^{\prime \prime}, \ldots, F^{(k)}$.

In particular we obtain an invariant of $f$, if we replace $F^{\prime}, F^{\prime \prime}, \ldots, F^{(k)}$ by $f$. Therefore

$$
\mathcal{I}=\sum c_{i_{1}, i_{2}, \ldots, i_{k}} A_{i_{1}} A_{i_{2}} \ldots A_{i_{k}}
$$

is an invariant of $f$.

Therefore we have demonstrated the following Theorem.

Theorem 9. Let $f$ be a $n$-form and let $d$ be an element of $H_{I}^{I n v}$ which is homogeneous of degree $n$ with respect to $1,2, \ldots, k$. Then the above construction gives an invariant $\mathcal{I}$ of $f$ of degree $k$.

Analogously, the symbolic method admits the construction of covariants, common invariants and common covariants.

The simplest symbols are $(12)^{2}$ and $(12)(13)(23)$ for the quadratic form, $(12)^{3}$ for the cubic form and $(12)^{4},(12)^{2}(13)^{2}(23)^{2}$ for the quartic form.

Example 6. We apply the symbolic method in the simplest example.

Let $f=x^{2} A+2 x y B+y^{2} C$ be a quadratic form. For the symbol $(12)^{2}$ we obtain

$$
\begin{gathered}
(12)^{2}=x_{1}^{2} y_{2}^{2}-2 x_{1} y_{1} x_{2} y_{2}+y_{1}^{2} x_{2}^{2}-h x_{1} y_{1} y_{2}^{2}+3 h y_{1}^{2} x_{2} y_{2} \\
=\left(C^{(1)}-3 h x_{1} y_{1}\right) y_{2}^{2}-2 x_{1} y_{1} x_{2} y_{2}+y_{1}^{2}\left(C^{(2)}-3 h x_{2} y_{2}\right)-h x_{1} y_{1} y_{2}^{2}+3 h y_{1}^{2} x_{2} y_{2} \\
=\left(C^{(1)}-3 h\left(-B^{(1)}-\frac{h}{2} y_{1}^{2}\right)\right) y_{2}^{2}-2\left(-B^{(1)}-\frac{h}{2} y_{1}^{2}\right)\left(-B^{(2)}-\frac{h}{2} y_{2}^{2}\right)
\end{gathered}
$$




$$
\begin{aligned}
& +y_{1}^{2}\left(C^{(2)}-3 h\left(-B^{(2)}-\frac{h}{2} y_{2}^{2}\right)\right)-h\left(-B^{(1)}-\frac{h}{2} y_{1}^{2}\right) y_{2}^{2}+3 h y_{1}^{2}\left(-B^{(2)}-\frac{h}{2} y_{2}^{2}\right) \\
& =\left(C^{(1)}-3 h\left(-B^{(1)}-\frac{h}{2} A^{(1)}\right)\right) A^{(2)}-2\left(-B^{(1)}-\frac{h}{2} A^{(1)}\right)\left(-B^{(2)}-\frac{h}{2} A^{(2)}\right) \\
& +A^{(1)}\left(C^{(2)}-3 h\left(-B^{(2)}-\frac{h}{2} A^{(2)}\right)\right)-h\left(-B^{(1)}-\frac{h}{2} A^{(1)}\right) A^{(2)}+3 h A^{(1)}\left(-B^{(2)}-\frac{h}{2} A^{(2)}\right) \\
& =A^{(1)} C^{(2)}+C^{(1)} A^{(2)}-2 B^{(1)} B^{(2)}+3 h B^{(1)} A^{(2)}-h A^{(1)} B^{(2)}+\frac{3 h^{2}}{2} A^{(1)} A^{(2)} .
\end{aligned}
$$

We identify the coefficients of $(01)^{2}$ and $(02)^{2}$ and obtain the discriminant

$$
d_{2}=A C+C A-2 B^{2}+3 h B A-h A B+\frac{3 h^{2}}{2} A^{2} .
$$

We can apply the commutation relations (11) in order to get the ordered expression

$$
d_{2}=2 A C-2 B^{2}-2 h A B+\frac{3 h^{2}}{2} A^{2}
$$

or the symmetric expression

$$
d_{2}=A C+C A-2 B^{2}+h(A B+B A)-\frac{5 h^{2}}{2} A^{2}
$$

or expressions without the term $A B$ or $A^{2}$

$$
\begin{aligned}
& d_{2}=\frac{3}{2} A C+\frac{1}{2} C A-2 B^{2}-\frac{3 h^{2}}{2} A^{2} \\
& =A C+C A-2 B^{2}-\frac{h}{4} A B+\frac{9 h}{4} B A .
\end{aligned}
$$

For the special forms $f=(01)(02)$ and $f=(01)^{2}$ we obtain $d_{2}=-\frac{1}{2}(12)^{2}$ and $d_{2}=0$, respectively. $(12)(13)(23)$ gives the zero invariant.

Example 7. Consider the cubic form $f$. The simplest symbol $(12)^{3}$ gives the zero invariant. The Hessian covariant $\Delta$ has the symbol $(01)(02)(12)^{2}$. Up to a constant factor we obtain

$$
\Delta=x^{2} K+x y L+y^{2} M
$$

with

$$
\begin{aligned}
& K=2 A C-2 B^{2}-4 h A B+2 h^{2} A^{2} \\
& L=2 A D-2 B C-2 h A C+4 h^{2} A B-2 h^{3} A^{2}, \\
& M=2 B D-2 C^{2}-4 h A D+6 h^{2} B^{2}-6 h^{3} A B+8 h^{4} A^{2} .
\end{aligned}
$$

The discriminant $d_{3}$ of $f$ has the symbol $(12)^{2}(34)^{2}(13)(24)$. Up to a scalar factor $d_{3}$ is also the discriminant of the quadratic form $\Delta$. We obtain

$$
2 d_{3}=2 K M-\frac{1}{2} L^{2}-h K L+\frac{3 h^{2}}{2} K^{2}
$$


and

$$
\begin{aligned}
& d_{3}=\quad-A^{2} D^{2}+6 A B C D-4 A C^{3}-4 B^{3} D+3 B^{2} C^{2} \\
& +9 h\left(-2 A B^{2} D+A B C^{2}-A^{2} C D\right)+h^{2}\left(-9 B^{4}+40 A^{2} B D-7 A^{2} C^{2}+12 A B^{2} C\right) \\
& +6 h^{3}\left(-12 A^{3} D-6 A^{2} B C-11 A B^{3}\right)+2 h^{4}\left(75 A^{2} B^{2}+38 A^{3} C\right) \\
& -384 h^{5} A^{3} B+652 h^{6} A^{4} \text {. }
\end{aligned}
$$

Furthermore the cubic covariant $j$ has the symbol $(01)^{2}(02)(13)(23)^{2}$. We have

$$
\begin{aligned}
j= & x^{3}\left(A^{2} D-3 A B C+2 B^{3}+9 h A B^{2}-2 h^{2} A^{2} B+6 h^{3} A^{3}\right) \\
+ & x^{2} y\left(+3 A B D-6 A C^{2}+3 B^{2} C-9 h A^{2} D+12 h A B C-3 h B^{3}-6 h^{2} A B^{2}-12 h^{3} A^{2} B+6 h^{4} A^{3}\right) \\
+ & x y^{2}\left(-3 A C D+6 B^{2} D-3 B C^{2}-6 h A B D+9 h A C^{2}-3 h B^{2} C\right. \\
& \left.+30 h^{2} A^{2} D-12 h^{2} A B C+12 h^{2} B^{3}-12 h^{3} A B^{2}+60 h^{4} A^{2} B-90 h^{5} A^{3}\right) \\
+ & y^{3}\left(-A D^{2}+3 B C D-2 C^{3}-15 h B^{2} D+6 h B C^{2}+36 h^{2} A B D-8 h^{2} A C^{2}+6 h^{2} B^{2} C\right. \\
& \left.-102 h^{3} A^{2} D-18 h^{3} A B C-42 h^{3} B^{3}+38 h^{4} A^{2} C+126 h^{4} A B^{2}-378 h^{5} A^{2} B+704 h^{6} A^{3}\right) .
\end{aligned}
$$

Remark 8. In the classical case, the zeros of $j$ are the three fourth harmonic points of the zeros of $f$ and the zeros of $\Delta$ are the two equiharmonic points of the zeros of $f$ or of $j$ (cf. [3]).

Remark 9. For $f, \Delta, j, d_{3}$ we have the syzygy

$$
4 \Delta^{3}+j^{2}+d_{3} f^{2}=0
$$

By Theorem 4 we can represent $f, \Delta, j, d_{3}$ by bracket symbols. The coefficients do not depend on $h$. By Corollary [6 the equation follows from the classical situation, cf. [3, 19].

\section{Polarization}

In this section we generalize the classical polarization operators $x_{l} \frac{\partial}{\partial x_{k}}+y_{l} \frac{\partial}{\partial y_{k}}$ by operators $\Delta_{k l}: H_{I} \rightarrow H_{I}$ with $k, l \in I$. We fix a PBW basis, which consists of the elements $x_{a_{1}}^{i_{1}} y_{a_{1}}^{j_{1}} x_{a_{2}}^{i_{2}} y_{a_{2}}^{j_{2}} \ldots x_{a_{k}}^{i_{k}} y_{a_{k}}^{j_{k}}$ with $a_{1}<a_{2}<\ldots<a_{k}$. We set

$$
\Delta_{k l}\left(x_{k}\right)=x_{l}, \quad \Delta_{k l}\left(y_{k}\right)=y_{l}
$$

and

$$
\Delta_{k l}\left(x_{i}\right)=0, \quad \Delta_{k l}\left(y_{i}\right)=0
$$

for $i \neq k$ and require that

$$
\Delta_{k l}(a b)=\Delta_{k l}(a) b+a \Delta_{k l}(b)
$$

for $a, b \in H_{I}$.

Proposition 10. $\Delta_{k l}$ is a well defined linear operator. 
Proof: One has to check the consistency of the Leibniz rule with the relations (5). For example we have

$$
\Delta_{k l}\left(y_{i} x_{k}\right)=y_{i} x_{l}=x_{l} y_{i}-h y_{l} y_{i}=\Delta_{k l}\left(x_{k} y_{i}-h y_{k} y_{i}\right) \cdot \bullet
$$

Furthermore we define the operator $P_{i j}: H_{I} \rightarrow H_{I}$ with

$$
P_{i j} a=\frac{1}{n} \Delta_{i j}(a)
$$

if $a$ is homogeneous of degree $n$ with respect to the index $i$. Analogous we define polarization operators $\Delta_{\left(x_{i}, y_{i}\right),(X, Y)}$ and $P_{\left(x_{i}, y_{i}\right),(X, Y)}$ where $X, Y$ are the homogeneous coordinates of a linear form. $P_{i j}$ and $P_{\left(x_{i}, y_{i}\right),(X, Y)}$ have the simple property

$$
P_{\left(x_{i}, y_{i}\right),(X, Y)}(i k)=(X k), \quad P_{\left(x_{i}, y_{i}\right),(X, Y)}(k l)=0
$$

for $i \neq k, l$ with $(X k):=X y_{k}-Y x_{k}-h Y y_{k} \in H_{I}^{I n v}$. In particular we have

$$
P_{i j}(i k)=(j k), \quad P_{i j}(k l)=0
$$

for $i \neq k, l$. We have the Leibniz rule

$$
P_{\left(x_{i}, y_{i}\right),(X, Y)}(a b)=n P_{\left(x_{i}, y_{i}\right),(X, Y)}(a) b+m a P_{\left(x_{i}, y_{i}\right),(X, Y)}(b)
$$

for $a, b \in H_{I}$ with $\operatorname{deg}_{i}(a)=m$ and $\operatorname{deg}_{i}(b)=n$. It follows

$$
P_{\left(x_{i}, y_{i}\right),(X, Y)} H_{I}^{I n v} \subseteq H_{I}^{I n v}
$$

Remark 10. Let $f$ be an element of $H_{I}$, let $x Y-y X-h y Y$ be a linear form and let $G=G_{\left(x_{i}, y_{i}\right),(X, Y)}$ be the algebra homomorphism which is determined by $G\left(x_{i}\right)=X$, $G\left(y_{i}\right)=Y$ and $G\left(x_{j}\right)=x_{j}, G\left(y_{j}\right)=y_{j}$ for $j \neq i$. Because of Proposition $8 G$ is compatible with (5). Therefore the algebra homomorphism $G$ is well defined. We can extend $G$ to $Q_{I}$.

Lemma 11. Let $f$ be a $k$-form and let $x Y-y X-h y Y$ be a linear form. If we replace $x, y$ in $f$ by $X, Y$, respectively, we obtain $P_{(x, y),(X, Y)}^{k} f$, i.e. we have $P_{(x, y),(X, Y)}^{k} f=G_{(x, y),(X, Y)} f$.

Proof: The Lemma is true in the classical case (cf. [3]). We represent $f$ by brackets $(i j)$. By (15), (17) the action of $P_{\left(x_{i}, y_{i}\right),(X, Y)}$ on the algebra of brackets is equal to the classical case. Therefore we obtain $P_{\left(x_{i}, y_{i}\right),(X, Y)}^{k} f$ if we replace all $(0 i)$ by $(X i)$. By Remark 10 the proposition follows.

Remark 11. An analogue of Lemma 11] is not true in the case of $U_{q}(s l(2))$-symmetry. For the noncommutative coordinate algebra in [12] we can define polarisation operators but we have no analogue of $G$.

Lemma 12. A formula which contains only brackets $(i j)$, polarization operators $P_{\left(x_{k}, y_{k}\right),(X, Y)}$ and complex coefficients is true if and only if this formula is valid in the classical case $h=0$.

Proof: The proof follows from (15), (17) and Corollary 6. • 
Example 8. By the corresponding classical result (cf. [3]) and Lemma 12 we obtain that $P_{01}^{n-1} f$ is a linear form with $P_{01}^{n-1} f=P_{10} P_{01}^{n} f$.

\section{Typical representation}

We will use the typical representation of a binary form (cf. [3, 7, 10, for the classical case) for the decomposition into linear forms. We consider the n-form

$$
f=x^{n} A_{0}+x^{n-1} y A_{1}+x^{n-2} y^{2} A_{2}+\cdots+y^{n} A_{n}
$$

with $A_{i} \in H_{I \backslash\{0,1\}}$ and the $n$-th polar

$$
f_{1}:=P_{01}^{n} f=x_{1}^{n} A_{0}+x_{1}^{n-1} y_{1} A_{1}+x_{1}^{n-2} y_{1}^{2} A_{2}+\cdots+y_{1}^{n} A_{n},
$$

cf. Lemma 11. Furthermore let $\xi, \eta$ be the two linear forms

$$
\begin{aligned}
\xi=P_{10} f_{1}=P_{01}^{n-1} f & =: \quad x \delta-y \gamma-h y \delta \\
\eta=(01) & =x y_{1}-y x_{1}-h y y_{1}
\end{aligned}
$$

with $\gamma, \delta \in H_{I \backslash\{0\}} \cdot \gamma, \delta$ have commutation relations analogous to $x_{i}, y_{i}$.

Theorem 13. We have

$$
f=f_{1}^{-n}\left(\xi^{n} u_{0}+\xi^{n-1} \eta u_{1}+\xi^{n-2} \eta^{2} u_{2}+\cdots+\eta^{n} u_{n}\right)
$$

with $u_{i} \in H_{I \backslash\{0\}}$. The coefficients $u_{0}, u_{1}, \cdots, u_{n}$ are covariants with respect to $x_{1}, y_{1}$.

Proof: We refer to [3] for the classical case. The Theorem follows from Lemma 12.

Remark 12. We call (19) the typical represetation of $f$. We mention that $u_{0}=f_{1}$ and $u_{1}=0$ (cf. [3]). One can derive the typical representation if one expresses $x, y$ by $\xi, \eta$. We have

$$
\begin{aligned}
\xi y_{1} & =x \delta y_{1}-y(\gamma+h \delta) y_{1} \\
\eta \delta & =x y_{1} \delta-y\left(x_{1}+h y_{1}\right) \delta
\end{aligned}
$$

Because of $y_{1} \delta=\delta y_{1}$ it follows

$$
\xi y_{1}-\eta \delta=y\left(x_{1} \delta-\gamma y_{1}\right)
$$

Furthermore we have

$$
\begin{aligned}
\xi x_{1} & =x \delta x_{1}-y(\gamma+h \delta) x_{1} \\
\eta \gamma & =x y_{1} \gamma-y\left(x_{1}+h y_{1}\right) \gamma
\end{aligned}
$$

Because of $\left(x_{1}+h y_{1}\right) \gamma=(\gamma+h \delta) x_{1}$ we have

$$
\xi x_{1}-\eta \gamma=x\left(\delta x_{1}-y_{1} \gamma\right)
$$

We mention the relation $\delta x_{1}-y_{1} \gamma=x_{1} \delta-\gamma y_{1}=x_{1} \delta-y_{1} \gamma-h y_{1} \delta=f_{1}$. From (20) and (21) we obtain the inverse transformation

$$
\begin{aligned}
& x=\left(\xi x_{1}-\eta \gamma\right) f_{1}^{-1}, \\
& y=\left(\xi y_{1}-\eta \delta\right) f_{1}^{-1} .
\end{aligned}
$$


We insert the expressions for $x, y$ into (18) and obtain

$$
f=f_{1}^{-n}\left(\left(\xi x_{1}-\eta \gamma\right)^{n} A_{0}+\left(\xi x_{1}-\eta \gamma\right)^{n-1}\left(\xi y_{1}-\eta \delta\right) A_{1}+\cdots+\left(\xi y_{1}-\eta \delta\right)^{n} A_{n}\right) .
$$

The elements $\xi, \eta$ are central. It follows

$$
f=f_{1}^{-n}\left(\xi^{n} c_{0}+\xi^{n-1} \eta c_{1}+\xi^{n-2} \eta^{2} c_{2}+\cdots+\eta^{n} c_{n}\right)
$$

with $c_{i} \in H_{I \backslash\{0\}}$. It is possible to show that $c_{i}=u_{i}$.

\section{The decomposition of binary forms into linear forms}

By (19) we can consider $f$ as a commutative $n$-form with variables $\xi, \eta \in Q_{I}^{I n v}$ and coefficients $u_{i} \in Q_{I \backslash\{0\}}^{I n v}$. It follows from Corollary [6 that there is no algebraic relation between $\xi, \eta$ and $u_{i}, i=1, \ldots, n$. Therefore there is a commutative splitting field $\Sigma \supset$ $Q_{I \backslash\{0\}}^{I n v}$ with

$$
f=C\left(\xi-\sigma_{1} \eta\right)\left(\xi-\sigma_{2} \eta\right) \cdots\left(\xi-\sigma_{n} \eta\right)
$$

where $\sigma_{i} \in \Sigma$ and $C=u_{0} f_{1}^{-n}=f_{1}^{1-n} \in Q_{I \backslash\{0\}}^{I n v}$.

We can suppose, that the elements $\sigma_{1}, \ldots, \sigma_{n}$ are invariant with respect to an extended action of $U_{h}(s l(2))$ and that they commute with the skew field $Q_{I}$.

Remark 13. In the classical case the elements $\sigma_{i}$ are the associated roots of G. Kohn (cf. [10]).

We consider the central skew field extensions $Q_{I \backslash\{0\}}^{\Sigma}:=Q_{I \backslash\{0\}}\left[\sigma_{1}, \ldots, \sigma_{n}\right]$ and $Q_{I}^{\Sigma}:=Q_{I}\left[\sigma_{1}, \ldots, \sigma_{n}\right]$, (cf. [4]). The elements $\left(\xi-\sigma_{i} \eta\right) \in Q_{I}^{\Sigma}$ are linear forms in $x, y$. We have

$$
\xi-\sigma_{i} \eta=x\left(\delta-\sigma_{i} y_{1}\right)-y\left(\gamma-\sigma_{i} x_{1}\right)-h y\left(\delta-\sigma_{i} y_{1}\right)=: x Y_{i}-y X_{i}-h y Y_{i}
$$

with $X_{i}=\gamma-\sigma_{i} x_{1}$ and $Y_{i}=\delta-\sigma_{i} y_{1} \in Q_{I \backslash\{0\}}^{\Sigma}$. Therefore we have

Theorem 14. Let $f=\sum_{i=1}^{n} x^{n-i} y^{i} A_{i}$ be a binary form with $A_{i} \in H_{I \backslash\{0,1\}}$. Then we have the decomposition

$$
f=C \prod_{i=1}^{n}\left(x Y_{i}-y X_{i}-h y Y_{i}\right)
$$

into (commuting, invariant) linear forms where $X_{i}, Y_{i}$ are elements of the central skew field extension $Q_{I \backslash\{0\}}^{\Sigma}$ and $C=f_{1}^{1-n} \in Q_{I \backslash\{0\}}^{I n v}$.

We can consider $X_{i}, Y_{i}$ as homogeneous coordinates of the unique $n$ zeros $Z_{i}=X_{i} Y_{i}^{-1}+\frac{h}{2}$ of $f$ in the following sense.

Proposition 15. Let $f$ be a n-form with the decomposition (24).

(i) We have $P_{(x, y),\left(X_{i}, Y_{i}\right)}^{n} f=0$.

(ii) Let $x Y-y X-h y Y$ be a linear form with $P_{(x, y),(X, Y)}^{n} f=0$. Then there is an $i \in$ $\{1,2, \ldots, n\}$ with $Z_{i}=X Y^{-1}+\frac{h}{2}$. 
Proof: (i) follows from Lemma 11 and $X_{i} Y_{i}-Y_{i} X_{i}-h Y_{i}^{2}=0$.

(ii) Let

$$
P_{(x, y),(X, Y)}^{n} f=C \prod_{i=1}^{n}\left(X Y_{i}-Y X_{i}-h Y Y_{i}\right)=0 .
$$

Because $Q_{I}^{\Sigma}$ has no zero divisors there is an $i$ with $X Y_{i}-Y X_{i}-h Y Y_{i}=0$. It follows $(X-h Y) Y_{i}=Y X_{i} \Longrightarrow Y^{-1} X-h=X_{i} Y_{i}^{-1} \Longrightarrow X Y^{-1}=X_{i} Y_{i}^{-1} \Longrightarrow X Y^{-1}+\frac{h}{2}=$ $X_{i} Y_{i}^{-1}+\frac{h}{2}$.

Remark 14. $X_{i}, Y_{i}$ and $C$ contain the additional variables $x_{1}, y_{1}$ which are not contained in the coefficients $A_{i}, i=0, \ldots, n$. It is possible to replace $x_{1}, y_{1}$ by the coefficients $X, Y$ of an arbitrary linear form with $P_{(x, y),(X, Y)} f \neq 0$. Then the analogous construction gives the points $X^{\prime}{ }_{i}, Y^{\prime}{ }_{i}$ with $X_{i}^{\prime} Y_{i}^{\prime-1}=X_{i} Y_{i}^{-1}$. Therefore $Z_{i}$ is independent of $x_{1}, y_{1}$.

In the following sections we will decompose the forms with $n=2,3$.

\section{The quadratic equation}

At first we consider the classical case. The classical quadratic form $f=a x^{2}+2 b x y+c y^{2}$ has the decomposition

$$
f=\frac{1}{P_{01}^{2} f}\left(x y_{+}-y x_{+}\right)\left(x y_{-}-y x_{-}\right)
$$

with

$$
x_{ \pm}=-b x_{1}-c y_{1} \pm x_{1} \sqrt{b^{2}-a c} \quad \text { and } \quad y_{ \pm}=a x_{1}+b y_{1} \pm y_{1} \sqrt{b^{2}-a c} .
$$

This decomposition corresponds to the solution of the quadratic equation $a z^{2}+2 b z+c=0$ of Clebsch

$$
z_{ \pm}=\frac{-b z_{1}-c \pm z_{1} \sqrt{b^{2}-a c}}{a z_{1}+b \pm \sqrt{b^{2}-a c}}
$$

(cf. [3, 16]).

Now let

$$
f=x^{2} A+2 x y B+y^{2} C
$$

be a noncommutative quadratic form with $A, B, C \in H_{I \backslash\{0,1\}}$ with the discriminant

$$
d_{2}=2 A C-2 B^{2}-2 h A B+\frac{3 h^{2}}{2} A^{2}
$$

We have the equation

$$
f P_{01}^{2} f=\left(P_{01} f\right)^{2}+\frac{1}{2} d_{2}(01)^{2}
$$

(typical representation of a quadratic form). This equation follows from the corresponding classical identity (cf. [3]) and Lemma [12.

It follows the decomposition into two linear forms

$$
\begin{aligned}
f P_{01}^{2} f= & \left(P_{01} f+(01) \sqrt{-\frac{1}{2} d_{2}}\right)\left(P_{01} f-(01) \sqrt{-\frac{1}{2} d_{2}}\right) \\
=: & \left(x Y_{1}-y X_{1}-h y Y_{1}\right)\left(x Y_{2}-y X_{2}-h y Y_{2}\right)
\end{aligned}
$$


We obtain for the homogeneous coordinates

$$
\begin{aligned}
X_{1,2} & :=-x_{1} B-y_{1} C \pm x_{1} \sqrt{-\frac{1}{2} d_{2}}-2 h y_{1} B-\frac{3}{2} h x_{1} A \\
Y_{1,2} & :=x_{1} A+y_{1} B \pm y_{1} \sqrt{-\frac{1}{2} d_{2}}-\frac{1}{2} h y_{1} A .
\end{aligned}
$$

For the calculations we used the central skew field extension

$$
Q_{I}\left[\sqrt{-\frac{1}{2} d_{2}}\right]=Q_{I}\left[\sqrt{B^{2}-A C+h A B-\frac{3 h^{2}}{4} A^{2}}\right] .
$$

For the corresponding projective coordinates $Z_{i}=X_{i} Y_{i}^{-1}+\frac{h}{2}, i=1,2$ we have

$$
Z_{1,2}:=X_{1,2} Y_{1,2}^{-1}+\frac{h}{2}=\left(-B \pm \sqrt{-\frac{1}{2} d_{2}}\right) A^{-1} .
$$

$Z_{1}, Z_{2}$ are obviously independent of $x_{1}, y_{1}$.

\section{The solution of the cubic equation}

Let

$$
f=x^{3} A+x^{2} y B+x y^{2} C+y^{3} D
$$

be a cubic form with the discriminant $d_{3}$, with the Hessian covariant $\Delta$ and with the cubic covariant $j$, cf. Example 7 Analogous to the classical case we have the equation

$$
f \cdot\left(P_{01}^{3} f\right)^{2}=\left(P_{01}^{2} f\right)^{3}+3 P_{01}^{2} \Delta \cdot(01)^{2} P_{01}^{2} f-P_{01}^{3} Q \cdot(01)^{3}
$$

(typical representation of a cubic form, cf. [3] and Lemma 12). With the notations $\Delta_{1}:=P_{01}^{2} \Delta, j_{1}:=P_{01}^{3} j, \xi=P_{01}^{2} f=P_{10} f_{1}$ and $\eta=(01)$ we have

$$
f f_{1}^{2}=\xi^{3}+3 \Delta_{1} \eta^{2} \xi-j_{1} \eta^{3}
$$

This is a commutative cubic form in $\xi$ and $\eta$ and we can apply the classical Cardano formula. We make the ansatz

$$
\xi \eta^{-1}=\varepsilon^{i} u_{1}+\varepsilon^{2 i} u_{2}
$$

with two commuting variables $u_{1}, u_{2}$ and $\varepsilon=e^{\frac{2 \pi i}{3}}$.

We insert (27) into (26). By Corollary [6 there are no algebraic relations between $\xi, \eta, \Delta_{1}, j_{1}$. Therefore a comparison of the coefficients yields

$$
\begin{aligned}
u_{1}^{3}+u_{2}^{3} & =j_{1}, \\
u_{1} u_{2} & =-\Delta_{1} .
\end{aligned}
$$

$u_{1}^{3}, u_{2}^{3}$ are the roots of the quadratic equation

$$
z^{2}-j_{1} z-\Delta_{1}^{3}=0
$$


It follows

$$
u_{1,2}^{3}=\frac{j_{1}}{2} \pm \sqrt{\frac{j_{1}^{2}}{4}+\Delta_{1}^{3}}
$$

Because of

$$
4 \Delta_{1}^{3}+j_{1}^{2}+d_{3} f_{1}^{2}=0
$$

(cf. Remark 91) we have

$$
u_{1,2}=\sqrt[3]{\frac{1}{2}\left(j_{1} \pm f_{1} \sqrt{-d_{3}}\right)}
$$

Therefore we have a decomposition into three linear forms

$$
f=C\left(\xi-\left(u_{1}+u_{2}\right) \eta\right)\left(\xi-\left(\varepsilon u_{1}+\varepsilon^{2} u_{2}\right) \eta\right)\left(\xi-\left(\varepsilon^{2} u_{1}+\varepsilon u_{2}\right) \eta\right)
$$

with $C \in Q_{I \backslash\{0\}}^{I n v}$. We have

$$
\xi-\left(\varepsilon^{i} u_{1}+\varepsilon^{2 i} u_{2}\right) \eta=x Y_{i}-y X_{i}-h y Y_{i}
$$

with

$$
\begin{aligned}
X_{i} & =\gamma-\left(\varepsilon^{i} u_{1}+\varepsilon^{2 i} u_{2}\right) x_{1}, \\
Y_{i} & =\delta-\left(\varepsilon^{i} u_{1}+\varepsilon^{2 i} u_{2}\right) y_{1}
\end{aligned}
$$

$(i=1,2,3) \cdot \gamma, \delta$ have the explizite form

$$
\begin{gathered}
\gamma=-x_{1}^{2}(B+h A)-x_{1} y_{1}\left(2 C+3 h B+h^{2} A\right)-y_{1}^{2}\left(D+2 h C+h^{2} B-h^{3} A\right), \\
\delta=x_{1}^{2} A+x_{1} y_{1}(2 B-h A)+y_{1}^{2}\left(C-h B+h^{2} A\right) .
\end{gathered}
$$

For the calculations we used the central skew field extension

$$
Q_{I}\left[\sqrt{-d_{3}}, \sqrt[3]{\frac{1}{2}\left(j_{1} \pm f_{1} \sqrt{-d_{3}}\right)}\right]
$$

For the projective coordinates $Z_{i}=X_{i} Y_{i}^{-1}+\frac{h}{2}, i=1,2,3$ we obtain

$$
Z_{i}=\left(\gamma-\left(\varepsilon^{i} u_{1}+\varepsilon^{2 i} u_{2}\right) x_{1}\right)\left(\delta-\left(\varepsilon^{i} u_{1}+\varepsilon^{2 i} u_{2}\right) y_{1}\right)^{-1}+\frac{h}{2}
$$

Remark 15. Without proof we mention an explizite formula for $Z_{i}$ independent of $x_{1}, y_{1}$. We extend $Q_{I}^{\Sigma}$ by the noncentral cubic root elements $w_{ \pm}=\sqrt[3]{\frac{1}{2}\left(S \pm A \sqrt{-d_{3}}\right)}$ where $S=A^{2} D-3 A B C+2 B^{3}+9 h A B^{2}-2 h^{2} A^{2} B+6 h^{3} A^{3}$ is the first coefficient of $j$. We require the commutation relations $w_{+} w_{-}=w_{-} w_{+}, w_{ \pm} y_{i}=y_{i} w_{ \pm}$and $w_{ \pm} x_{i}=x_{i} w_{ \pm}-\frac{1}{3} h y_{i} w_{ \pm}$. Then we have

$$
Z_{i}=-B A^{-1}+\frac{3}{2} h+\epsilon^{i} A^{-1} \sqrt[3]{\frac{1}{2}\left(S+A \sqrt{-d_{3}}\right)}+\epsilon^{2 i} A^{-1} \sqrt[3]{\frac{1}{2}\left(S-A \sqrt{-d_{3}}\right)} .
$$


Remark 16. The quartic form has the typical representation

$$
f f_{1}^{3}=\xi^{4}+3 \Delta_{1} \xi^{2} \eta^{2}+T_{1} \xi \eta^{3}+\left(\frac{P f_{1}^{2}}{2}-\frac{3 \Delta_{1}^{2}}{4}\right) \eta^{4}
$$

with the Hessian covariant $\Delta$ of degree 4, the skew covariant $T$ of degree 6 and the fundamental invariant $P$ of order 2 (cf. [3]).

\section{The total differential and differential modules}

In the following we introduce differentials and differential modules. Consider the algebra $H_{I}$. We fix a subset $K \subset I$ with $0 \in K$. The elements $x_{i}, y_{i}(i \in K)$ play the role of variables, the elements $x_{i}, y_{i}$ with $i \in I \backslash K$ are constant with respect to our derivation $d$.

We define the differential module $\Gamma_{I, K}=Q_{I} d_{K} Q_{I}$. (Analogously one can consider differential modules $H_{I} d H_{I}$.) $\Gamma_{I, K}$ is a $Q_{I}$-module whose elements are linear combinations of monomials

$$
a d x_{i}, \quad b d y_{i}, \quad d x_{i} \cdot c, \quad d y_{i} \cdot d
$$

with $a, b, c, d \in Q_{I}, i \in K$. (The differentials $d x_{i}=d_{K} x_{i}, d y_{i}=d_{K} y_{i}$ with $i \in K$ are abstract elements). The defining relations of the $Q_{I}$-module are

$$
\begin{gathered}
d(\alpha a+\beta b)-\alpha d a-\beta d b=0, \\
d(a b)-d a \cdot b-a d b=0 \\
d 1=0
\end{gathered}
$$

for $a, b \in Q_{I}, \alpha, \beta \in \mathbb{C}$ and

$$
\begin{array}{ll}
d x_{j} \cdot x_{i}-x_{i} d x_{j}+h x_{i} d y_{j}-h y_{i} d x_{j}-h^{2} y_{i} d y_{j}=0, & d y_{j} \cdot y_{i}-y_{i} d y_{j}=0 \\
d x_{j} \cdot y_{i}-y_{i} d x_{j}-h y_{i} d y_{j}=0, & d y_{j} \cdot x_{i}-x_{i} d y_{j}+h y_{i} d y_{j}=0
\end{array}
$$

for $i \in I, j \in k$.

These relations allow us to represent every element of $\Gamma_{I, K}$ in the form

$$
\sum_{i \in I} a_{i} d x_{i}+b_{i} d y_{i}
$$

with $a_{i}, b_{i} \in Q_{I}$.

We define the total differential $d=d_{K}$ as a linear map $d_{K}: Q_{I} \rightarrow \Gamma_{I, K}$ by

$$
\begin{array}{ccl}
d_{K}\left(x_{i}\right)=d x_{i}, & d_{K}\left(y_{i}\right)=d y_{i} & \forall i \in K, \\
d_{K}\left(x_{i}\right)=0, & d_{K}\left(y_{i}\right)=0 & \forall i \in I \backslash K
\end{array}
$$

and we require the Leibniz rule

$$
d_{K}(a b)=d_{K} a \cdot b+a d_{K} b
$$

for $a, b \in Q_{I}$.

Proposition 16. $d_{K}$ is a well defined linear map. 
Proof: One has to check the consistency of the Leibniz rule with the relations (5). E.g. we have for $i, j \in K$

$$
d\left(x_{j} y_{i}\right)=x_{j} d y_{i}+d x_{j} \cdot y_{i}=d y_{i} \cdot x_{j}+h d y_{i} \cdot y_{j}+y_{i} d x_{j}+h y_{i} d y_{j}=d\left(y_{i} x_{j}+h y_{i} y_{j}\right) \bullet
$$

Remark 17. We identify $K$ with a set $K^{\prime}$ with $I \cap K^{\prime}=0$. Because of the similarity of the commutation relations (28) and (5) we can identify $\Gamma_{I, K}$ with a linear subspace of $Q_{I \cup K^{\prime}}$ according to $d x_{i} \cong x_{i^{\prime}}$ and $d y_{i} \cong y_{i^{\prime}}$.

Because of the identification in Remark 17] we can consider $\Gamma_{I, K}$ as a left $U_{h}(\operatorname{sl}(2))$ module. The action is given by

$$
\begin{array}{lll}
E d x_{i}=0, & F d x_{i}=d y_{i}, & H d x_{i}=d x_{i}, \\
E d y_{i}=d x_{i}, & F d y_{i}=0, & H d y_{i}=-d y_{i}
\end{array}
$$

for $i \in K$ and by an extension of the Leibniz rules (4) to $\Gamma_{I, K}$.

Proposition 17. $d_{K}$ commutes with the left $U_{h}(s l(2))$-action.

Proof: By (30) the proposition is valid for the generators $x_{i}, y_{i} \in H_{I}$. We suppose the proposition for $a, b \in H_{I}$ with $\operatorname{deg}(a), \operatorname{deg}(b) \leq n$. Because of $d e^{h F}(a)=e^{h F}(d a)$ for $a \in H_{I}$ we have

$$
\begin{aligned}
& E d(a b)=E(d a \cdot b+a d b)=E(d a) e^{-h F}(b)+e^{h F}(d a) E(b)+E(a) e^{-h F}(d b)+e^{h F}(a) E(d b) \\
& =d E(a) e^{-h F}(b)+d e^{h F}(a) E(b)+E(a) d e^{-h F}(b)+e^{h F}(a) d E(b)=d\left(E(a) e^{-h F}(b)+e^{h F}(a) E(b)\right)=d E(a b) .
\end{aligned}
$$

Similar we obtain $d F=F d, d H=H d$. The proposition follows.

Definition 4. We call $\gamma \in \Gamma_{I, K}$ a differential invariant, if $E \gamma=F \gamma=H \gamma=0$. We denote the submodule of differential invariants by $\Gamma_{I, K}^{I n v}$.

By Proposition [17 we have

$$
d_{K}\left(Q_{I}^{I n v}\right) \subseteq \Gamma_{I, K}^{I n v} .
$$

For $i \in K, j \in I \backslash K$ we obtain the differential invariant

$$
d_{K}((i j))=x_{i} d y_{j}-y_{i} d x_{j}-h y_{i} d y_{j} .
$$

We will use the notation $(i d j)=x_{i} d y_{j}-y_{i} d x_{j}-h y_{i} d y_{j}$.

We need the following simple Lemma about the structure of differential modules.

Lemma 18. Let $K, K^{\prime} \subset I$ and $K \cap K^{\prime}=\emptyset$. Then we have $\Gamma_{I, K \cup K^{\prime}}=\Gamma_{I, K} \oplus \Gamma_{I, K^{\prime}}$ and $d_{K \cup K^{\prime}}=d_{K}+d_{K^{\prime}}$.

The proof follows from the Leibniz rule.

Remark 18. Differential modules $S d_{K} S$ for central field extensions $S=Q_{I}\left[\sigma_{1}, \ldots, \sigma_{k}\right]$ are defined analogous to $Q_{I} d Q_{I}$. Additional requirements are the defining relations of the $\sigma_{i}$ and the conditions $\sigma_{i} d x-d x \cdot \sigma_{i}=x d \sigma_{i}-d \sigma_{i} \cdot x=0$ for $x \in S$. 
We can extend the polarization operators $P_{i j}$ and $P_{\left(x_{i}, y_{i}\right),(X, Y)}$ to $H_{I} d H_{I}$. The extensions are given if we set $P_{i j}(d a):=d P_{i j}(a), P_{\left(x_{i}, y_{i}\right),(X, Y)}(d a):=d P_{\left(x_{i}, y_{i}\right),(X, Y)}(a)$ and require that the Leibniz rule (17) is valid for $a, b \in H_{I} d H_{I}$. By Remark 17 the extended operators are well defined.

\section{Polynomials}

In this section we consider polynomials associated to $n$-forms.

Lemma 19. The elements $y_{i},(i j)$ and $z_{i}-z_{j}$ generate a commutative subfield of $Q_{I}$.

This follows immediately from $y_{i} y_{j}=y_{j} y_{i}, z_{i}-z_{j}=(i j) y_{i}^{-1} y_{j}^{-1}$ and Proposition 3 .

We introduce the bracket

$$
[i j):=y_{i}^{-1}(i j)=(i j) y_{i}^{-1}=\left(z_{i}-\frac{h}{2}\right) y_{j}-x_{j}
$$

with the properties $[i j][k l)=[k l)[i j)$ and $[i j) y_{k}=y_{k}[i j)$ for all $i, j, k, l$. Let $f=$ $(01)(02) \ldots(0 n)$ be a $n$-form. We introduce the the polynomial $f_{z}$ of $f$ by

$$
f_{z}:=y^{-n} f=[01)[02) \ldots[0 n)=\prod_{i=1}^{n}\left(\left(z-\frac{h}{2}\right) y_{i}-x_{i}\right) .
$$

By polarization we obtain

$$
f_{Z}:=Y^{-n} P_{(x, y),(X, Y)}^{n} f=[Z 1)[Z 2) \ldots[Z n)=\prod_{i=1}^{n}\left(\left(Z-\frac{h}{2}\right) y_{i}-x_{i}\right)
$$

with $Z:=X Y^{-1}+\frac{h}{2}$ and $[Z i):=\left(Z-\frac{h}{2}\right) y_{i}-x_{i}$.

We consider the differential module $\Gamma_{I, K}=Q_{I} d_{K} Q_{I}$. We have

$$
d_{i}[i j)=y_{j} d z_{i} \quad \text { if } \quad i \in K, j \notin K
$$

and

$$
d_{j}[i j)=\left(z_{i}-\frac{h}{2}\right) d y_{j}-d x_{j}=:[0 d j) \quad \text { if } \quad i \notin K, j \in K .
$$

Furthermore we have

$$
d z=d x \cdot y^{-1}+x d y^{-1}=d x \cdot y^{-1}-x y^{-2} d y=(d x \cdot y-d y \cdot x-h d y \cdot y) y^{-2}=-(0 d 0) y^{-2} .
$$

We generalize Lemma 19]

Lemma 20. The elements $y_{i}, z_{i}-z_{j},(i j),[i j), d y_{i}, d z_{i},(i d j),[i d j)$ commute in $\Gamma_{I, K}$.

We compute the differentials of $f_{z}$. Let $K=\{0,1,2, \ldots, n\}$ and $d=d_{0}+\delta=d_{0}+d_{\{1,2, \ldots, n\}}$.

Lemma 21. We have

$$
d_{0} f_{z}=d_{0}([01)[02) \ldots[0 n))=\left(\sum_{i=1}^{n} y_{i}[01) \ldots[0, i-1)[0, i+1) \ldots[0 n)\right) d z
$$

and

$$
\delta f_{z}=\delta([01)[02) \ldots[0 n))=\sum_{i=1}^{n}[01) \ldots[0, i-1)[0 d i)[0, i+1) \ldots[0 n)
$$




\section{Elliptic and Hyperelliptic Differentials}

We extend $Q_{I}$ by the central element $w$ with

$$
w^{2}=(01)(02)(03)(04) .
$$

We obtain the central skew field extension $Q_{I}[w]$. We denote $w$ by

$$
w=\sqrt{(01)(02)(03)(04)} .
$$

We can suppose that $w$ is invariant.

Definition 5. We say that the differential invariant

$$
d e_{x, y}:=w^{-1} y^{2} d z=\sqrt{(01)(02)(03)(04)}^{-1} \quad(y d x-x d y+h y d y)
$$

of the differential module $Q_{I}[w] d_{K} Q_{I}[w]$ with $0 \in K$ and $1,2,3,4 \notin K$ is an elliptic differential of the first kind.

Remark 19. In the classical case we have

$$
d e_{x, y}=\frac{y d x-x d y}{\sqrt{\left(x y_{1}-y x_{1}\right)\left(x y_{2}-y x_{2}\right)\left(x y_{2}-y x_{2}\right)\left(x y_{2}-y x_{2}\right)}}=\frac{\frac{1}{\sqrt{y_{1} y_{2} y_{3} y_{4}}} d z}{\sqrt{\left(z-z_{1}\right)\left(z-z_{2}\right)\left(z-z_{3}\right)\left(z-z_{4}\right)}} .
$$

More generally, for $g \in \mathbb{N}, g \geq 1$ we extend $Q_{I}$ by the central and invariant element $W$ with

$$
W^{2}=\left(0 i_{1}\right) \cdots\left(0 i_{2 g+2}\right)
$$

where the indices $i_{j}$ are different. We obtain the central skew field extension $Q_{I}[W]$. We use the notation

$$
W=\sqrt{\left(0 i_{1}\right) \cdots\left(0 i_{2 g+2}\right)} .
$$

Furthermore we consider the $(g-1)$-form

$$
U=\left(0 j_{1}\right) \cdots\left(0 j_{g-1}\right) .
$$

Definition 6. We say that the differential invariant

$$
\begin{gathered}
d h_{x, y}:=U W^{-1} y^{2} d z \\
=\left(0 j_{1}\right) \cdots\left(0 j_{g-1}\right) \quad{\sqrt{\left(0 i_{1}\right) \cdots\left(0, i_{2 g+2}\right)}}^{-1} \quad(y d x-x d y-h y d y)
\end{gathered}
$$

of the differential module $Q_{I}[W] d_{K} Q_{I}[W]$ with $0 \in K$ and $i_{1}, \ldots, i_{2 g+2}, j_{1}, \ldots, j_{g-1} \notin K$ is a hyperelliptic differential of the first kind.

Remark 20. In the classical case we have

$$
\begin{gathered}
d h_{x, y}=\frac{\left(x y_{j_{1}}-y x_{j_{1}}\right) \cdots\left(x y_{j_{g-1}}-y x_{j_{g-1}}\right)(y d x-x d y)}{\sqrt{\left(x y_{i_{1}}-y x_{i_{1}}\right)\left(x y_{i_{2}}-y x_{i_{2}}\right) \ldots \ldots \ldots \ldots \ldots\left(x y_{i_{2 g+2}}-y x_{i_{2 g+2}}\right)}} \\
=\frac{y_{j_{1}} \ldots y_{j_{g-1}}}{\sqrt{y_{i_{1}} \cdots y_{i_{2 g+2}}}} \frac{\left(z-z_{j_{1}}\right) \cdots\left(z-z_{j_{g-1}}\right) d z}{\sqrt{\left(z-z_{i_{1}}\right)\left(z-z_{i_{2}}\right) \ldots \ldots \ldots \ldots\left(z-z_{i_{2 g+2}}\right)}} .
\end{gathered}
$$

$d h_{x, y}$ is connected with an algebraic curve of genus $g$. In particular for $g=1$ we obtain elliptic differentials. The $g$ classical hyperelliptic differentials of the first kind $\frac{z^{i} d z}{\sqrt{p(z)}}$, $i=0,1,2, \ldots, g-1$ correspond to $d h_{x, y}$ with $U=\left(0 j_{1}\right)^{i}\left(0 j_{2}\right)^{g-1-i}$, where $\left(x_{j_{1}}, y_{j_{1}}\right)$ is the zero point and $\left(x_{j_{2}}, y_{j_{2}}\right)$ is the point at infinity. 
We can extend the polarization process $P_{(x, y),(X, Y)}$ to extensions of fields and of differential modules. We change the arguments of elliptic and hyperelliptic differentials by the definition

$$
d e_{X, Y}:=\left(\sqrt{P_{(x, y),(X, Y)}^{4} w^{2}}\right)^{-1} P_{(x, y),(X, Y)}^{2}(d 0,0)
$$

and

$$
d h_{X, Y}:=\left(\sqrt{P_{(x, y),(X, Y)}^{2 g+2} W^{2}}\right)^{-1} P_{(x, y),(X, Y)}^{g+1}(U(d 0,0)) .
$$

for arbitrary noncommutative points $(X, Y)$ with $Z=X Y^{-1}+\frac{h}{2}$.

Remark 21. We obtain $d e_{X, Y}$ and $d h_{X, Y}$ by replacing $(x, y)$ by $(X, Y)$ in $d e_{x, y}$ and $d h_{x, y}$, respectively.

\section{The Addition theorem and Abel's Theorem}

Let $\Gamma_{I, K}$ be a differential module with $1,2,3,4 \notin K$ and $0,5 \in K$.

For the above elliptic differential (30) we consider the cubic form

$$
r=(01)(02)(03)-(04)(05)^{2} .
$$

According to Theorem 14 we have the decomposition into commuting factors

$$
r=C\left(x Y_{1}-y X_{1}-h y Y_{1}\right)\left(x Y_{2}-y X_{2}-h y Y_{2}\right)\left(x Y_{3}-y X_{3}-h y Y_{3}\right)
$$

with $X_{i}, Y_{i}$ in a splitting field $Q_{I \backslash\{0\}}^{\Sigma}$ and $C \in Q_{I \backslash\{0\}}^{I n v}$. We have $r_{X_{i}, Y_{i}}:=P_{(x, y),\left(X_{i}, Y_{i}\right)}^{3} r=0$ for $i=1,2,3$.

Furthermore we extend $Q_{I}^{\Sigma}$ by the central root elements

$$
w^{(i)}:=\sqrt{P_{(x, y),(X, Y)}^{4} w^{2}}=\sqrt{\left(X_{i} 1\right)\left(X_{i} 2\right)\left(X_{i} 3\right)\left(X_{i} 4\right)}, \quad i=1,2,3
$$

with $\left(X_{i} j\right):=X_{i} y_{j}-Y_{i} x_{j}-h Y_{i} y_{j}$ to the skew field $Q_{I}^{\Sigma, w}$.

Now we consider the elliptic differentials

$$
d e_{X_{i}, Y_{i}}:=\left(w^{(i)}\right)^{-1}\left(d X_{i}, X_{i}\right)
$$

of the differential module $\Gamma_{I, K}:=Q_{I}^{\Sigma, w} d_{K} Q_{I}^{\Sigma, w}$.

We formulate the addition theorem.

Theorem 22. Let $\left(X_{i}, Y_{i}\right)$ be the homogeneous coordinates of the linear factors of $r=$ $(01)(02)(03)-(04)(05)^{2}$. Then we have

$$
\epsilon_{1} d e_{X_{1}, Y_{1}}+\epsilon_{2} d e_{X_{2}, Y_{2}}+\epsilon_{3} d e_{X_{3}, Y_{3}}=0
$$

with certain $\epsilon_{i}= \pm 1$.

Remark 22. In the classical case the Theorem reduces to the differential equation

$$
\frac{d Z_{1}}{\sqrt{\left(Z_{1}-z_{1}\right)\left(Z_{1}-z_{2}\right)\left(Z_{1}-z_{3}\right)\left(Z_{1}-z_{4}\right)}}+\frac{d Z_{2}}{\sqrt{\left(Z_{2}-z_{1}\right)\left(Z_{2}-z_{2}\right)\left(Z_{2}-z_{3}\right)\left(Z_{2}-z_{4}\right)}}+\frac{d Z_{3}}{\sqrt{\left(Z_{3}-z_{1}\right)\left(Z_{3}-z_{2}\right)\left(Z_{3}-z_{3}\right)\left(Z_{3}-z_{4}\right)}}=0
$$

if $Z_{1}, Z_{2}, Z_{3}$ vary continuously as the zeros of the equations

$$
\left(z-z_{1}\right)\left(z-z_{2}\right)\left(z-z_{3}\right)-\left(z-z_{4}\right)(a z+b)^{2}=0
$$


with arbitrary $a, b \in \mathbb{C}$. We can consider $Z_{1}, Z_{2}, Z_{3}$ as the coordinates of the intersection points of the lines $q(z, u, a, b)=u-a z-b=0$ and the elliptic curve $p(z, u)=u^{2}\left(z-z_{4}\right)-\left(z-z_{1}\right)\left(z-z_{2}\right)\left(z-z_{3}\right)=0$ or as the intersection points of the variable curve $q(z, u, a, b)=(a z+b) u-\left(z-z_{1}\right)\left(z-z_{2}\right)\left(z-z_{3}\right)=0$ and the elliptic curve $p(z, u)=u^{2}-\left(z-z_{1}\right)\left(z-z_{2}\right)\left(z-z_{3}\right)\left(z-z_{4}\right)=0$. If we choose two different points with coordinates $Z_{1}, Z_{2}$ and $\epsilon_{1}, \epsilon_{2}$ then the parameters $a, b$ are fixed and $Z_{3}, \epsilon_{3}$ are uniquely determined (classical addition theorem).

For the consideration of hyperelliptic differentials we suppose $i_{1}, i_{2}, \cdots, i_{2 g+2}, j_{1}, \cdots, j_{g-1} \notin$ $K$ and $0, k_{1}, \cdots, k_{p}, l_{1}, \cdots, l_{q} \in K$. We require that the indices $i_{j}, k_{j}, l_{j}$ are different (cf. below for $k_{j}, l_{j}$ ).

For the above hyperelliptic differential we fix a number $s=0,1, \ldots, 2 g+2$ and two forms $P=\left(0 k_{1}\right) \ldots\left(0 k_{p}\right), Q=\left(0 l_{1}\right) \ldots\left(0 l_{q}\right)$ of degree $p$ and $q$, respectively, with

$$
p-q=g+1-s
$$

It follows

$$
s+2 p=2 g+2-s+2 q .
$$

Remark 23. The equation $p-q=g+1-s$ is a technical condition for this invariant theoretical consideration in order to secure the homogeneity of $r$ (cf. below). Furthermore one can replace $P$ and $Q$ by arbitrary $p$ - and $q$-forms, respectively.

Furthermore we consider the decomposition

$$
W^{2}=A B
$$

with $A=\left(0 i_{1}\right) \ldots\left(0 i_{s}\right)$ and $B=\left(0, i_{s+1}\right) \ldots\left(0, i_{2 g+2}\right)$. Therefore

$$
r:=\left(0 i_{1}\right) \ldots\left(0 i_{s}\right) P^{2}-\left(0, i_{s+1}\right) \ldots\left(0, i_{2 g+2}\right) Q^{2}=A P^{2}-B Q^{2}
$$

is the difference of two $k$-forms with $k:=s+2 p=2 g+2-s+2 q=p+q+g+1$.

According to Theorem [14 we have the decomposition

$$
r=C\left(0 X_{1}\right) \cdots\left(0 X_{k}\right)
$$

into commuting factors $\left(0 X_{i}\right):=x Y_{i}-y X_{i}-h y Y_{i}$ with $X_{i}, Y_{i}$ in a splitting field $Q_{I \backslash\{0\}}^{\Sigma}$ and $C \in Q_{I \backslash\{0\}}^{I n v}$. We have $r_{X_{i}, Y_{i}}=P_{(x, y),\left(X_{i}, Y_{i}\right)}^{k} r=0$ for $i=1, \cdots k$.

Furthermore we extend $Q_{I}^{\Sigma}$ by the root elements

$$
W^{(i)}:=\sqrt{P_{(x, y),\left(X_{i}, Y_{i}\right)}^{2 g+2} W^{2}}=\sqrt{\left(X_{i} i_{1}\right) \cdots\left(X_{i} i_{2 g+2}\right)}
$$

to $Q_{I}^{\Sigma, W}$.

Then we consider the differential module $\Gamma_{I, K}=Q_{I}^{\Sigma, W} d_{K} Q_{I}^{\Sigma, W}$ and the hyperelliptic differentials

$$
d h_{X_{i}, Y_{i}}=W^{(i)}{ }^{-1} P_{(x, y),\left(X_{i}, Y_{i}\right)}^{g-1} U\left(d X_{i}, X_{i}\right)
$$

with $i=1,2, \ldots, k$. We formulate Abel's Theorem: 
Theorem 23. Let $\left(X_{i}, Y_{i}\right), i=1,2, \ldots, k$ be the homogeneous coordinates of the linear factors of $r=\left(0 i_{1}\right) \ldots\left(0 i_{s}\right) P^{2}-\left(0, i_{s+1}\right) \ldots\left(0, i_{2 g+2}\right) Q^{2}$. Then we have

$$
\epsilon_{1} d h_{X_{1}, Y_{1}}+\ldots .+\epsilon_{k} d h_{X_{k}, Y_{k}}=0
$$

with certain $\epsilon_{i}= \pm 1$.

Remark 24. In the classical case we have

$$
\sum_{i=1}^{k} \frac{U_{z}\left(Z_{i}\right) d Z_{i}}{\sqrt{\left(Z_{i}-z_{1}\right)\left(Z_{i}-z_{2}\right) \ldots\left(Z_{i}-z_{2 g+2}\right)}}=0
$$

where $Z_{1}, \ldots, Z_{k}$ vary continuously as coordinates of the intersection points of the curves $c\left(z, u, a_{0}, \ldots, a_{q}, b_{0}, \ldots, b_{p}\right)=\left(a_{0}+a_{1} z+\ldots+a_{q} z^{q}\right) u-\left(b_{0}+b_{1} z+\ldots+b_{p} z^{p}\right)\left(z-z_{1}\right) \ldots(z-$ $\left.z_{s}\right)=0$ and the hyperelliptic curve $p(z, u)=u^{2}-\left(z-z_{1}\right)\left(z-z_{2}\right) \ldots\left(z-z_{2 g+2}\right)=0$. A point of $p(u, z)=0$ is given by a pair $(Z, \epsilon)$ with $\epsilon= \pm 1$. We choose different points $\left(Z_{1}, \epsilon_{1}\right), \ldots,\left(Z_{p+q+1}, \epsilon_{p+q+1}\right)$. Then we obtain a system of the $p+q+1$ linear equations

$$
c\left(Z_{i}, \epsilon_{i} \sqrt{\left(Z_{i}-z_{1}\right) \ldots\left(Z_{i}-z_{2 g+2}\right)}, a_{0}, \ldots, a_{q}, b_{0}, \ldots, b_{p}\right)=0
$$

for the $\mathrm{p}+\mathrm{q}+2$ coefficients $a_{0}, \ldots, b_{p}$. In the generic case the rank is $p+q+1$ and the coefficients are determined up to a common factor. In this non-degenerate case the remaining $g$ points $\left(Z_{i}, \epsilon_{i}\right)$ are uniquely determined.

\section{Proof of Theorem 23}

We prove Theorem 23. We obtain Theorem 22 as the special case $g=1, s=3, p=0$, (i.e. $q=1, k=3, W=w, A=(01)(02)(03), B=(04), U=1, P=1, Q=(05))$.

Using invariant theory, the the proof is analogous to the classical case (cf. [1, 22]). We consider the $k$-form $r=A P^{2}-B Q^{2}$ with the decomposition $r=C\left(x Y_{1}-y X_{1}-h y Y_{1}\right) \ldots\left(x Y_{k}-y X_{k}-h y Y_{k}\right)$ with $X_{i}, Y_{i}$ in a splitting field $Q_{I \backslash\{0\}}^{\Sigma}$ and $C \in Q_{I \backslash\{0\}}^{I n v}$ and with $r_{X_{i}, Y_{i}}=P_{(x, y),\left(X_{i}, Y_{i}\right)}^{k} r=0$ for $i=1, \ldots, k$.

In order to apply $d=d_{K}$ we introduce $r_{z}$. We have

$$
r_{z}=y^{-k} r=C\left[0 X_{1}\right) \ldots\left[0 X_{k}\right)=\prod_{i=1}^{k}\left(\left(z-\frac{h}{2}\right) Y_{i}-X_{i}\right) .
$$

We consider the differential $d=d_{0}+\delta:=d_{0}+d_{\left\{k_{1}, \ldots k_{p}, l_{1}, \ldots, l_{q}\right\}}$ (cf. Lemma 18). We obtain

$$
d r_{z}=d_{0} r_{z}+\delta r_{z}=d_{0} r_{z}+2 A_{z} P_{z} \delta P_{z}-2 B_{z} Q_{z} \delta Q_{z}
$$

with

$$
\begin{gathered}
d_{0} r_{z}=\sum_{i=1}^{k} C Y_{i}\left[0 X_{1}\right) \ldots\left[0 X_{i-1}\right)\left[0 X_{i+1}\right) \ldots\left[0 X_{k}\right) d z \\
\delta P_{z}=\sum_{i=1}^{p}\left[0 k_{1}\right) \ldots\left[0 k_{i-1}\right)\left[0 d k_{i}\right)\left[0 k_{i+1}\right) \ldots\left[0 k_{p}\right)
\end{gathered}
$$

and

$$
\delta Q_{z}=\sum_{i=1}^{q}\left[0 l_{1}\right) \ldots\left[0 l_{i-1}\right)\left[0 d l_{i}\right)\left[0 l_{i+1}\right) \ldots\left[0 l_{q}\right)
$$


cf. Lemma 21. We multiply both sides with $y^{k+1}=y^{p+q+g+2}$. Using $y \circ \delta=\delta \circ y$ we obtain

$$
y^{k+1} d r_{z}=y^{k+1} d_{0} r_{z}+2 y A P \delta P-2 y B Q \delta Q
$$

with

$$
\begin{aligned}
y^{k+1} d_{0} r_{z} & =\sum_{i=1}^{k} C Y_{i}\left(0 X_{1}\right) \ldots\left(0 X_{i-1}\right)\left(0 X_{i+1}\right) \ldots\left(0 X_{k}\right)(d 0,0) \\
\delta P & =\sum_{i=1}^{p}\left(0 k_{1}\right) \ldots\left(0 k_{i-1}\right)\left(0 d k_{i}\right)\left(0 k_{i+1}\right) \ldots\left(0 k_{p}\right)
\end{aligned}
$$

and

$$
\delta Q=\sum_{i=1}^{q}\left(0 l_{1}\right) \ldots\left(0 l_{i-1}\right)\left(0 d l_{i}\right)\left(0 l_{i+1}\right) \ldots\left(0 l_{q}\right) .
$$

We apply $P_{(x, y),\left(X_{i}, Y_{i}\right)}^{k+1}$ to (32). Because of $r_{X_{i}, Y_{i}}=0$ we obtain for the left side

$$
P_{(x, y),\left(X_{i}, Y_{i}\right)}^{k+1}\left(y^{k+1} d\left(y^{-k} r\right)\right)=P_{(x, y),\left(X_{i}, Y_{i}\right)}^{k+1}(-k d y \cdot r+y d r)=-k d Y_{i} \cdot r_{X_{i}, Y_{i}}+Y_{i} d r_{X_{i}, Y_{i}}=0 .
$$

Therefore we have for the right side

$$
\begin{aligned}
0= & C Y_{i}\left(X_{i} X_{1}\right) \ldots\left(X_{i} X_{i-1}\right)\left(X_{i} X_{i+1}\right) \ldots\left(X_{i} X_{k}\right)\left(d X_{i}, X_{i}\right)+ \\
& 2 Y_{i} A_{X_{i}, Y_{i}} P_{X_{i}, Y_{i}} \delta P_{X_{i}, Y_{i}}-2 Y_{i} B_{X_{i}, Y_{i}} Q_{X_{i}, Y_{i}} \delta Q_{X_{i}, Y_{i}}
\end{aligned}
$$

We have for $i=1,2, \ldots, k$ the identity

$$
r_{X_{i}, Y_{i}}=P_{(x, y),\left(X_{i}, Y_{i}\right)}^{k} r=0
$$

It follows

$$
\begin{gathered}
A_{X_{i}, Y_{i}} P_{X_{i}, Y_{i}}^{2}-B_{X_{i}, Y_{i}} Q_{X_{i}, Y_{i}}^{2}=0 \\
A_{X_{i}, Y_{i}}^{2} P_{X_{i}, Y_{i}}^{2}-\left(W^{(i)}\right)^{2} Q_{X_{i}, Y_{i}}^{2}=0, \\
\left(A_{X_{i}, Y_{i}} P_{X_{i}, Y_{i}}+W^{(i)} Q_{X_{i}, Y_{i}}\right)\left(A_{X_{i}, Y_{i}} P_{X_{i}, Y_{i}}-W^{(i)} Q_{X_{i}, Y_{i}}\right)=0 .
\end{gathered}
$$

Because the skew field $Q_{I}^{\Sigma, W}$ has no zero divisors it follows

$$
A_{X_{i}, Y_{i}} P_{X_{i}, Y_{i}}=\epsilon_{i} W^{(i)} Q_{X_{i}, Y_{i}}
$$

with $\epsilon_{i}=1$ or -1 . From (34) and (35) it follows

$$
B_{X_{i}, Y_{i}} Q_{X_{i}, Y_{i}}=\epsilon_{i} W^{(i)} P_{X_{i}, Y_{i}} .
$$

We multiply (33) with $Y_{i}^{-1}$ and we insert the relations (35) and (36).

$C\left(X_{i} X_{1}\right) \ldots\left(X_{i} X_{i-1}\right)\left(X_{i} X_{i+1}\right) \ldots\left(X_{i} X_{k}\right)\left(d X_{i}, X_{i}\right)=-2 \epsilon_{i} W^{(i)} Q_{X_{i}, Y_{i}} \delta P_{X_{i}, Y_{i}}+2 \epsilon_{i} W^{(i)} P_{X_{i}, Y_{i}} \delta Q_{X_{i}, Y_{i}}$.

Because $i_{j}, k_{j}, l_{j}$ are different indices, $r$ has $k$ different zeros. Therefore we have $\left(X_{i} X_{j}\right) \neq 0 \forall i, j \in\{1, \ldots, k\}$. It follows

$\epsilon_{i} W^{(i)^{-1}} Y_{i}^{2} d Z_{i}=-2 C^{-1}\left(\left(X_{i} X_{1}\right) \ldots\left(X_{i} X_{i-1}\right)\left(X_{i} X_{i+1}\right) \ldots\left(X_{i} X_{k}\right)\right)^{-1}\left(Q_{X_{i}, Y_{i}} \delta P_{X_{i}, Y_{i}}-P_{X_{i}, Y_{i}} \delta Q_{X_{i}, Y_{i}}\right)$. 
We multiply both sides with the polars $U_{X_{i}, Y_{i}}=P_{(x, y),\left(X_{i}, Y_{i}\right)}^{g-1} U$ of an arbitrary $(g-1)$-form $U$.

$$
\begin{gathered}
\epsilon_{i} d h_{X_{i}, Y_{i}}=\epsilon_{i} U_{X_{i}, Y_{i}} W^{(i)}{ }^{-1} Y_{i}^{2} d Z_{i}= \\
-2 C^{-1}\left(\left(X_{i} X_{1}\right) \ldots\left(X_{i} X_{i-1}\right)\left(X_{i} X_{i+1}\right) \ldots\left(X_{i} X_{k}\right)\right)^{-1} U_{X_{i}, Y_{i}}\left(Q^{(i)} \delta P_{X_{i}, Y_{i}}-P^{(i)} \delta Q_{X_{i}, Y_{i}}\right) .
\end{gathered}
$$

We have to show that

$$
\begin{gathered}
\sum_{i=1}^{k} \epsilon_{i} d h_{X_{i}, Y_{i}}= \\
-2 C^{-1} \sum_{i=1}^{k}\left(\left(X_{i} X_{1}\right) \ldots\left(X_{i} X_{i-1}\right)\left(X_{i} X_{i+1}\right) \ldots\left(X_{i} X_{k}\right)\right)^{-1} U_{X_{i}, Y_{i}}\left(Q^{(i)} \delta P_{X_{i}, Y_{i}}-P^{(i)} \delta Q_{X_{i}, Y_{i}}\right)=0,
\end{gathered}
$$

where

$$
\delta P_{X_{i}, Y_{i}}=\sum_{j=1}^{p}\left(X_{i} k_{1}\right) \ldots\left(X_{i} k_{j-1}\right)\left(X_{i} d k_{j}\right)\left(X_{i} k_{j+1}\right) \ldots\left(X_{i} k_{p}\right)
$$

and

$$
\delta Q_{X_{i}, Y_{i}}=\sum_{j=1}^{q}\left(X_{i} l_{1}\right) \ldots\left(X_{i} l_{j-1}\right)\left(X_{i} d l_{j}\right)\left(X_{i} l_{j+1}\right) \ldots\left(X_{i} l_{q}\right) .
$$

The proposition follows from the following Lemma.

Lemma 24. Let $f=\left(0 X_{1}\right) \ldots\left(0 X_{k}\right)$ be a $k$-form with different zeros (i.e. $Z_{i} \neq Z_{j}$ for $i \neq j$ ) and let $g$ be $a(k-2)$-form. Then we have

$$
\sum_{i=1}^{k} \frac{P_{(x, y),\left(X_{i}, Y_{i}\right)}^{k-2} g}{\left(X_{i} X_{1}\right) \ldots\left(X_{i} X_{i-1}\right)\left(X_{i} X_{i+1}\right) \ldots\left(X_{i} X_{k}\right)}=0 .
$$

Proof: The form $g$ has a decomposition $g=C\left(0 X^{\prime}{ }_{1}\right) \ldots\left(0 X^{\prime}{ }_{k-2}\right)$ with bracket symbols. At first we consider the classical case $h=0$. Let $f_{z}(z)=y^{-k} f(x, y)$ and $g_{z}(z)=y^{-k+2} g(x, y)$. We have the partial fraction expansion

$$
\frac{-z g_{z}(z)}{f_{z}(z)}=\sum_{i=1}^{k} \frac{-Z_{i} g_{z}\left(Z_{i}\right)}{f_{z}^{\prime}\left(Z_{i}\right)\left(z-Z_{i}\right)}
$$

For $z=0$ we obtain

$$
\begin{gathered}
0=\sum_{i=1}^{k} \frac{g_{z}\left(Z_{i}\right)}{f^{\prime}{ }_{z}\left(Z_{i}\right)}=\sum_{i=1}^{k} \frac{g_{z}\left(Z_{i}\right)}{Y_{i}\left[Z_{i} X_{1}\right) \ldots\left[Z_{i} X_{i-1}\right)\left[Z_{i} X_{i+1}\right) \ldots\left[Z_{i} X_{k}\right)} \\
=\sum_{i=1}^{k} \frac{P_{(x, y),\left(X_{i}, Y_{i}\right)}^{k-2} g}{\left(X_{i} X_{1}\right) \ldots\left(X_{i} X_{i-1}\right)\left(X_{i} X_{i+1}\right) \ldots\left(X_{i} X_{k}\right)} .
\end{gathered}
$$

In the special case $X_{i}=x_{\alpha_{i}}, Y_{i}=y_{\alpha_{i}}, X^{\prime}{ }_{i}=x_{\beta_{i}}, Y^{\prime}{ }_{i}=y_{\beta_{i}}$ with different $\alpha_{i}, \beta_{i} \in I$ the Lemma follows from Lemma 12. In the general case we apply the field homomorphism with $x_{\alpha_{i}} \rightarrow X_{i}, y_{\alpha_{i}} \rightarrow Y_{i}, x_{\beta_{i}} \rightarrow X^{\prime}{ }_{i}, y_{\beta_{i}} \rightarrow Y^{\prime}{ }_{i}, x_{k} \rightarrow x_{k}$ and $y_{k} \rightarrow y_{k}$ for $k \neq \alpha_{i}, \beta_{i}$.

We identify the differentials $d x_{k_{i}}, d y_{k_{i}}, d x_{l_{i}}, d y_{l_{i}}$ with the coordinates $x_{m_{i}}, y_{m_{i}}, x_{n_{i}}, y_{n_{i}}$, respectively, with $m_{i}, n_{i} \notin I$, cf. Remark 17. Therefore we can consider $U(Q \delta P-P \delta Q)$ as a $(k-2)$-form $g$. Furthermore let $f$ be the $k$-from $r$. The Theorem follows from Lemma 24. 


\section{References}

1. Abel, N.H.: Remarques sur quelques proprietes generales d'une certain sorte de fonctions transcendantes, J. Reine Angew. Math. 3 (1828) 313-323, or in: Oeuvres, ed. by Sylow and Lie, Christiania 1881, T.1, 444-456.

2. Clebsch, A. and Gordan, P.: Theorie der Abelschen Funktionen, Teubner-Verlag, Leipzig 1866.

3. Clebsch, A.: Theorie der binären algebraischen Formen, Teubner, Leipzig, 1872.

4. Cohn, P.M., Skew fields, Cambridge University Press, 1995.

5. Curtis, C.W.: A note on noncommutative polynomials, Proc. Amer. Math. Soc. 3 (1952), 965-969.

6. Harris, J.: Algebraic geometry: a first course, Springer-Verlag, Berlin 1992.

7. Hermite, Ch.: Sur la theorie des fonctions homogenes a deux indeterminees, J. Reine Angew. Math. 52 (1866), 18-38.

8. Kassel, C.: Quantum Groups, Springer Verlag, New York, 1995.

9. Klimek, S. and Lesniewski, A.: A Two-Parameter Quantum Deformation of the Unit Disc, J. Func. Anal. 115 (1993), 1-23.

10. Kohn, G.: Zur Theorie der associirten Formen, Wien. Ber. C. 1891, 865-893.

11. Kontsevich, M.: Deformation quantisation of algebraic varieties, Preprint AG/0106006.

12. Leitenberger, F.: A Quantum Deformation of Invariants of Higher Binary Forms, J. Algebra 222 (1999), 82-128.

13. Majid, S.: Foundations of Quantum Group Theory, Cambridge University Press, New York, 1995.

14. Manin, Y.: Quantized Theta functions, Progress of Theor. Phys. Supplement, 102 (1990) 219-228.

15. McConnel, J.C. and Robson, J.C.: Noncommutative Noetherian Rings, John Wiley \& Sons, Chichester, 1987.

16. Matthiessen, L.: Grundzüge der antiken und modernen Algebra der litteralen Gleichungen, Teubner, Leipzig, 1878.

17. Mumford, D.: Tata lectures on theta II, Birkhäuser, Boston 1994.

18. Ore, O.: Linear equations in non-commutative fields, Ann. Math. 32 (1933), 480-508.

19. Olver, P.: Classical Invariant theory, Cambridge University Press, Cambridge, 1999.

20. Stafford, J.T. and Van den Bergh M.: Noncommutative curves and noncommutative surfaces, Bull. AMS 38(2001)171-216. 
21. Sturmfels, B.: Algorithms in Invariant Theory, Springer-Verlag, Wien, 1993.

22. Weber, H.: Elliptische Funktionen und algebraische Zahlen, Vieweg, Braunschweig, 1908. 